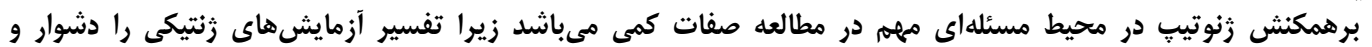

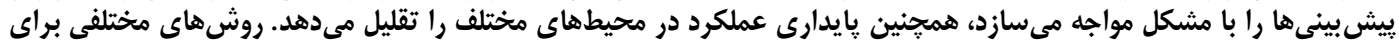

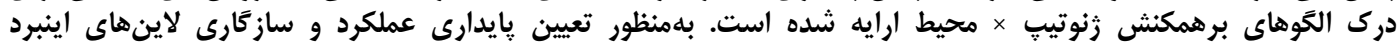

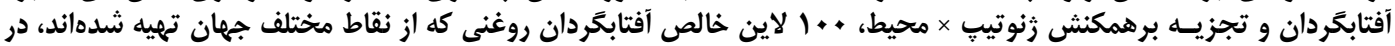

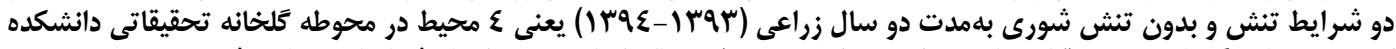

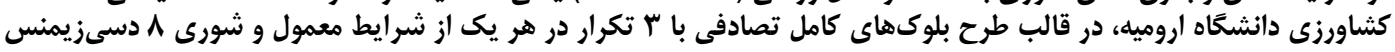

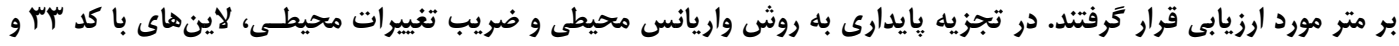

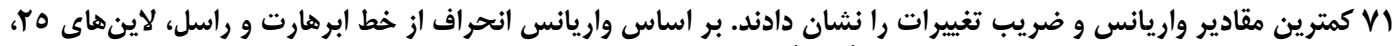

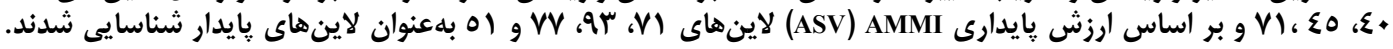

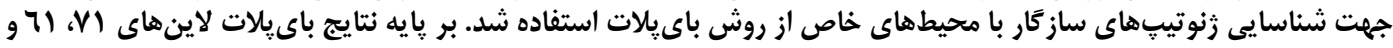

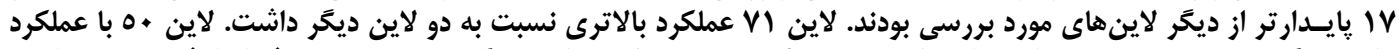

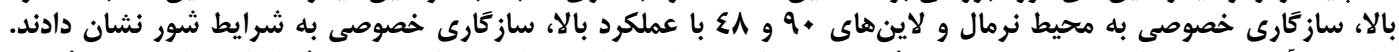

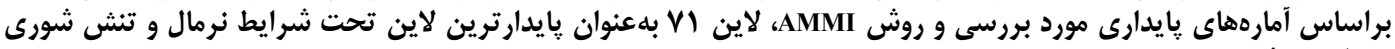

معرفى مى شود.

وازههاى كليدى: آفتابكردان، برهمكنش رنوتيب × محيط، باى يلات، تنش شورى

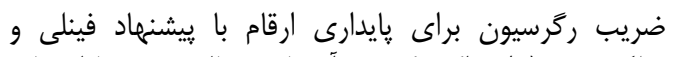

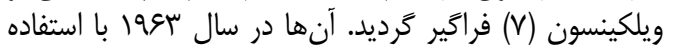

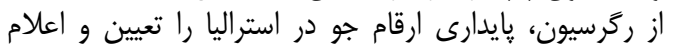

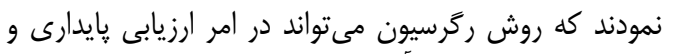

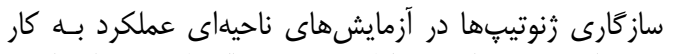

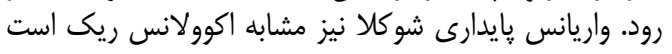

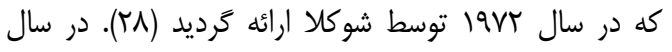

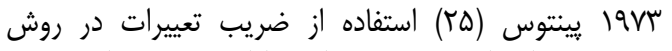

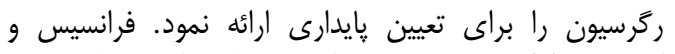

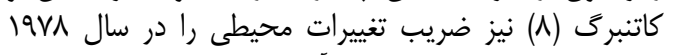

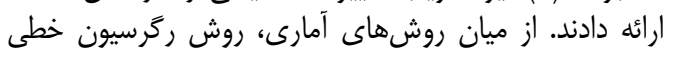

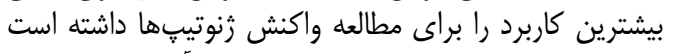

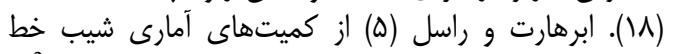

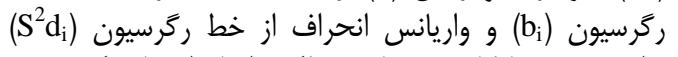

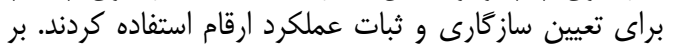

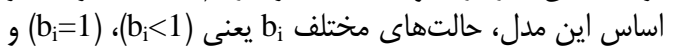

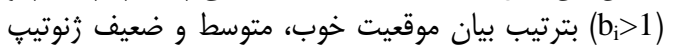

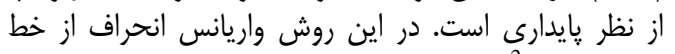
ركر إيون (S

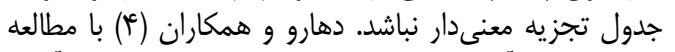

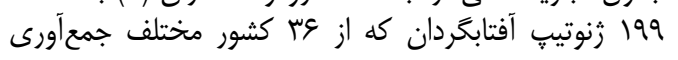

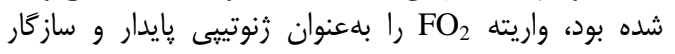

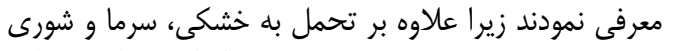

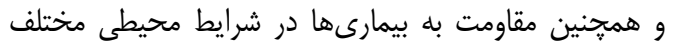

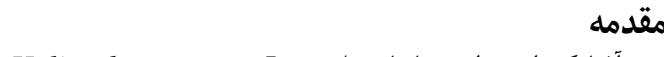

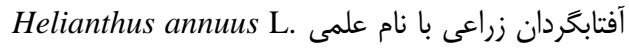

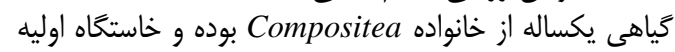

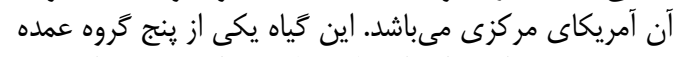

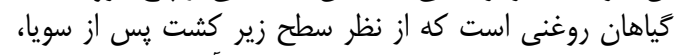

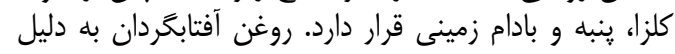

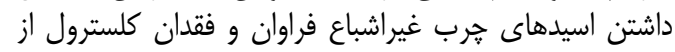

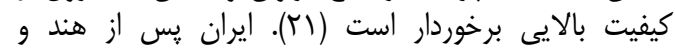

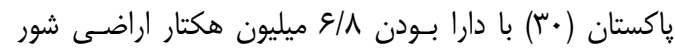

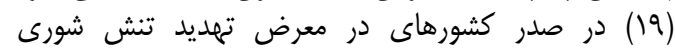

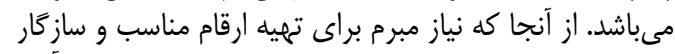

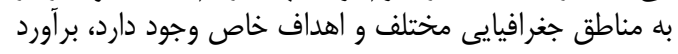

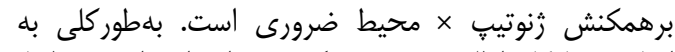

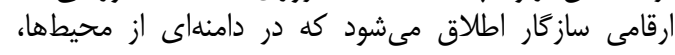

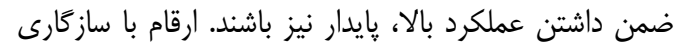

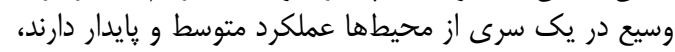

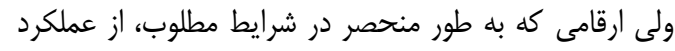

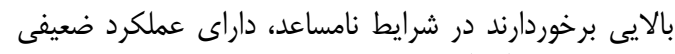

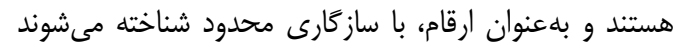

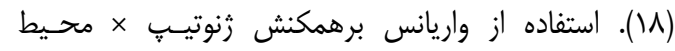

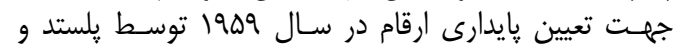

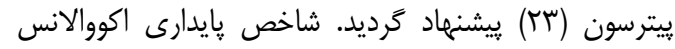

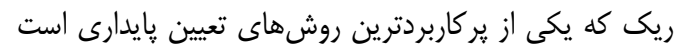

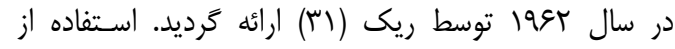




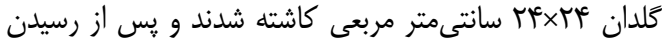

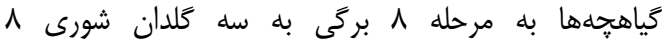

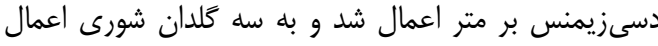

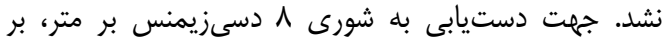

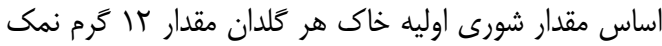
NaCl

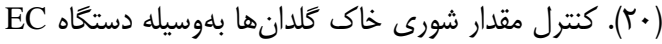

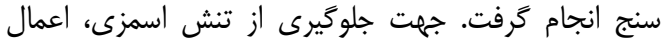

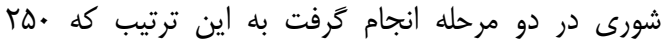

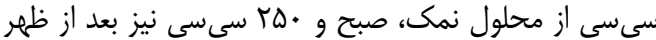

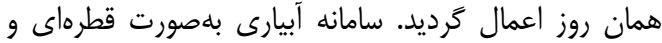

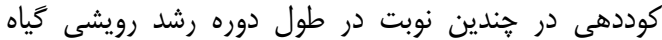

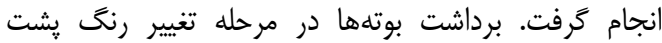

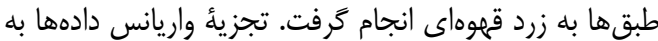

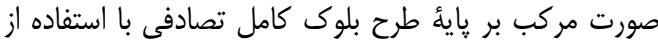

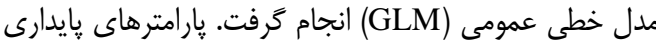

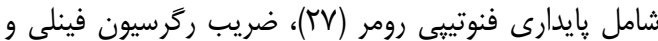

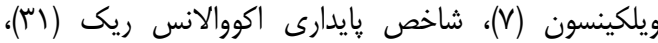

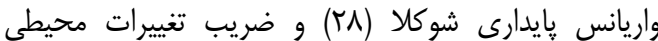

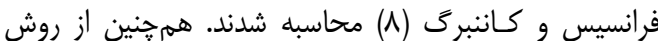

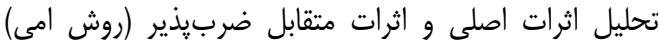

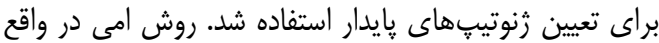

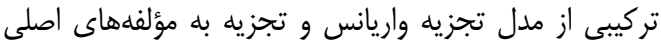
$Y_{\mathrm{ijk}}=\mu+\mathrm{g}_{\mathrm{i}}+\mathrm{e}_{\mathrm{j}}+\Sigma \delta_{\mathrm{n}} \zeta_{\mathrm{in}} \eta_{\mathrm{jn}}$ است و مدل

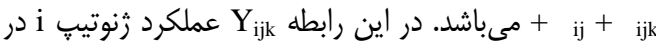

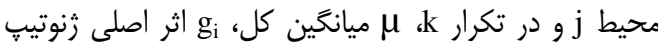

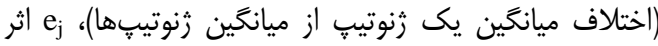

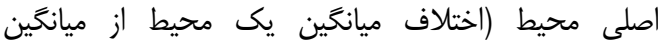

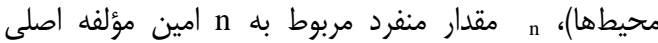

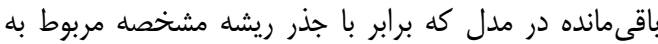

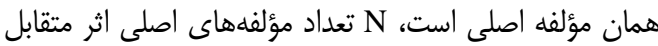

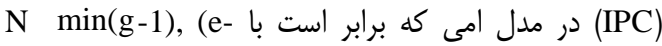

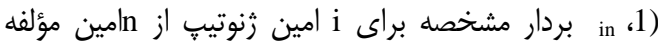

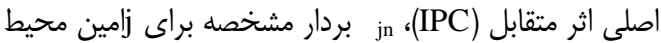

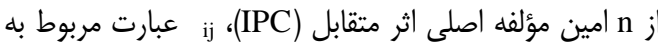

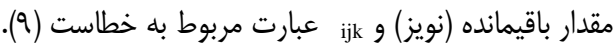

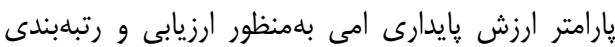

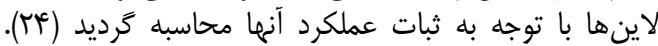

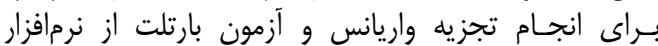

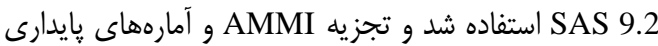

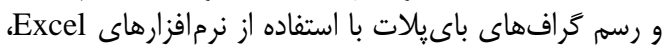
SAS 9.2

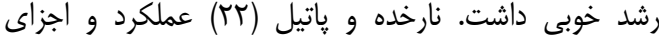

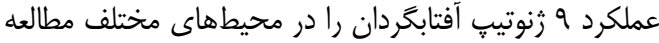

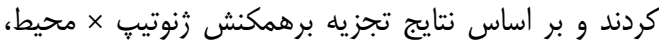

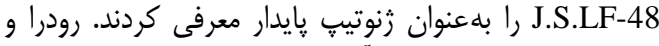

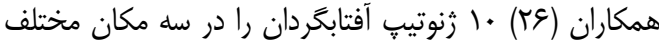

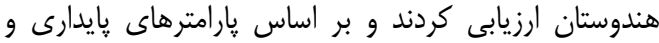
ميانخين عملكرد، زنوتيبٍ هاى

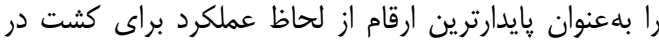

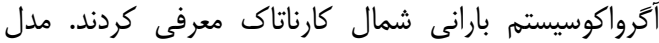

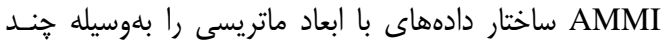

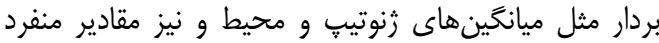

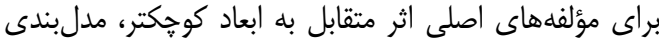

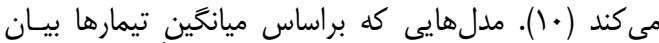

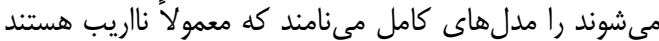

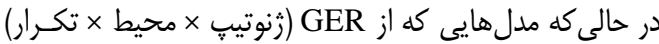

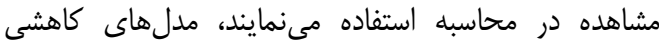

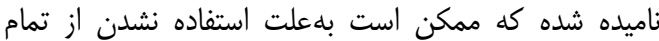

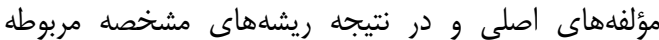

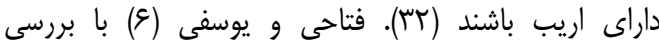

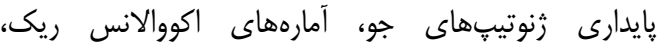

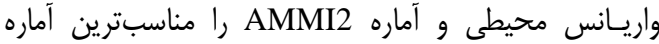

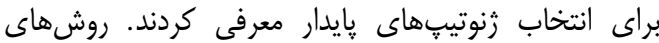

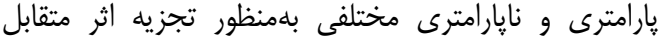

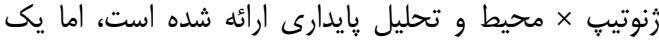

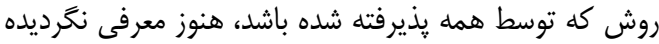

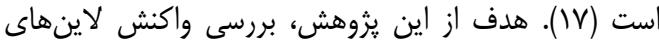

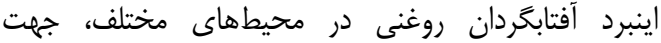

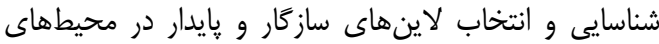

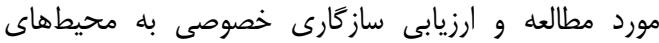

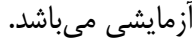

\section{مواد و روشها}

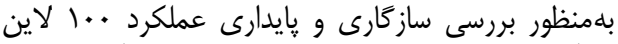

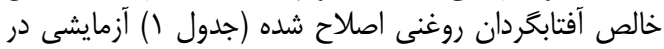

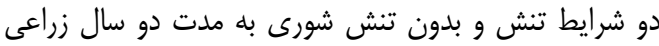

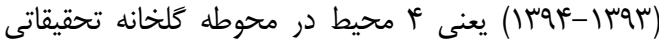

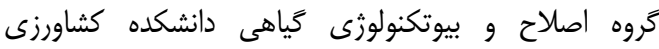

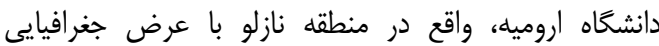

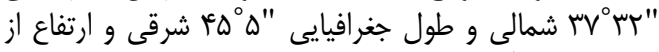

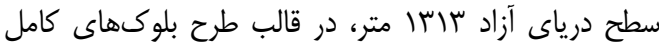

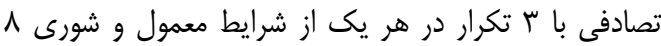

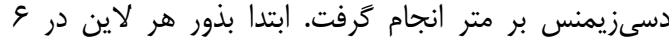




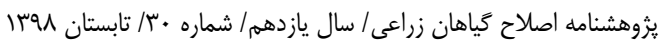

جدول 1- مشخصات لاينهاى آفتابخردان روغنى مورد مطالعه در شرايط نرمال و تنش شورى Table 1. Characteristics of oily sunflower lines studied under normal and salt stress conditions

\begin{tabular}{|c|c|c|c|c|c|c|c|}
\hline نام مركز تحقيقاتى & ن ام كشور & نام لاين & كد & نام مركز تحقيقاتى & ن ام كشور & نام لاين & كد \\
\hline USDA & امريكا & RHA265 & D) & ASGROW & فرانسه & H100A/83HR4 & 1 \\
\hline USDA & امريكا & PM1-3 & ar & ASGROW & فرانسه & H209A/LC1064 & r \\
\hline RUSTICA & فرانسه & RT948 & $\Delta r$ & ASGROW & فرانسه & H205A/H543R & r \\
\hline- & - & 283-ENSAT & $\Delta f$ & ENSAT & فرانسه & AS5306 & r \\
\hline INRAMONT & فرانسه & QHP-1 & $\Delta \Delta$ & USDA & امريكا اما & RHA858 & $\Delta$ \\
\hline USDA & امريكا & SDR19 & $\Delta F$ & ASGROW & فرانسه & H209A/83HR4 & 8 \\
\hline USDA & امريكا & HА337B & $\Delta V$ & ENSAT & فرانسه & AS3211 & v \\
\hline ASGROW & فرانسه & H100B & $\Delta \wedge$ & ENSAT & فرانسه & 254-ENSAT & $\wedge$ \\
\hline- & مجارستان & B454/03 & $\Delta 9$ & ASGROW & فرانسه & AS5304 & 9 \\
\hline USDA & امريكا & HA304 & 9. & ENSAT & فرانسه & $1009329.2(100 \mathrm{~K})$ & 1. \\
\hline RUSTICA & فرانسه & RT931 & 9) & ENSAT & فرانسه & 270-ENSAT & 11 \\
\hline USDA & امريكا & НА335B & gr & ASGROW & فرانسه & AS613 & ir \\
\hline NOVARTIS & فرانسه & NS_B5 & r & NOVARTIS & فرانسه & A-F1POPA & سו \\
\hline USDA & امريكا & SDB3 & $a t^{c}$ & INRAMONT & فرانسه & OES & If \\
\hline ASGROW & فرانسه & LC1064C & $9 \Delta$ & ASGROW & فرانسه & H100A/LC1064 & 10 \\
\hline NOVARTIS & فرانسه & NS-R5 & 99 & USDA & امريكا & RHA266 & 19 \\
\hline USDA & امريكا & DM-2 & gV & ENSAT & فرانسه & PAC2 & iv \\
\hline ASGROW & فرانسه & H156A/RHA274 & 91 & ASGROW & فرانسه & H157A/LC1064 & i \\
\hline USDA & امريكا & SDB1 & 99 & BRN & فرانسه & 5DES20QR & 19 \\
\hline USDA & امريكا & HAR-4 & v. & ENSAT & فرانسه & $1009337(100 \mathrm{~K})$ & r. \\
\hline ASGROW & فرانسه & AS5305 & $n$ & ENSAT & فرانسه & AS3232 & r \\
\hline USDA & امريكا & RHA274 & vT & ASGROW & فرانسه & 12ASB3 & r \\
\hline ASGROW & فرانسه & $\mathrm{H} 158 \mathrm{~A} / \mathrm{H} 543 \mathrm{R}$ & $v^{\mu}$ & ASGROW & فرانسه & 8ASB2 & r \\
\hline ASGROW & فرانسه & H100A/RHA274 & $v^{e}$ & Caussade semences & فرانسه & $9 \mathrm{CSA} 3$ & re \\
\hline ASGROW & فرانسه & $\mathrm{H} 209 \mathrm{~A} / \mathrm{H} 566 \mathrm{R}$ & Va & - & فرانسه & $\mathrm{H} 049+\mathrm{FSB}$ & ro \\
\hline ENSAT & فرانسه & ASO-1-POP-A & ve & ASGROW & فرانسه & SSD-580 & צ \\
\hline ENSAT & فرانسه & AS6305 & w & ASGROW & فرانسه & $5 \mathrm{AS}-\mathrm{F}_{1} / \mathrm{A}_{2} * \mathrm{R}_{2}$ & rV \\
\hline NOVARTIS & فرانسه & B-F1POPB & vı & C.F & فرانسه & 7CR16=PRH6 & ru \\
\hline USDA & امريكا & D34 & va & ENSAT & فرانسه & ENSAT699 & ra \\
\hline ENSAT & فرانسه & CAY & $\Lambda$. & ASGROW & فرانسه & SSD-581 & r. \\
\hline SPII & ايران & 346 & $\wedge 1$ & INRAMONT & فرانسه & TMB-51 & ו \\
\hline NOVARTIS & فرانسه & $\mathrm{NS}-\mathrm{F}_{1}-\mathrm{A}_{5} * \mathrm{R}_{5}$ & Ar & SPII & ايران & $10-59$ & Tr \\
\hline SPII & ايران & 36 & ^r & SPII & ايران & 110 & זس \\
\hline SPII & ايران & 38 & Af & INRAMONT & فرانسه & H603R & re \\
\hline INRAMONT & فرانسه & SDB2 & $\Lambda \Delta$ & SPII & ايران & 4 & ra \\
\hline- & - & H158A/LC1064 & N & ENSAT & فرانسه & 703-CHLORINA & q \\
\hline ASGROW & فرانسه & $\mathrm{H} 156 \mathrm{~A} / \mathrm{H} 543 \mathrm{R}$ & $\wedge \vee$ & NOVARTIS & فرانسه & $\mathrm{NSF}_{1}-\mathrm{A}_{4} * \mathrm{R}_{5}$ & r \\
\hline ASGROW & فرانسه & $\mathrm{H} 543 \mathrm{R} / \mathrm{H} 543 \mathrm{R}$ & $M$ & SPII & ايران & 28 & н \\
\hline- & فرانسه & H543R & 19 & SPII & ايران & 30 & rq \\
\hline ASGROW & فرانسه & 15038 & q. & - & مجارستان & F1250/03 & r. \\
\hline ENSAT & فرانسه & SF076 & 91 & USDA & امريكا & SDR18 & il \\
\hline- & - & $8 \mathrm{~A} * \mathrm{LC} 1064 \mathrm{C} *$ & q5 & ENSAT & فرانسه & LP-CSYB & et \\
\hline ENSAT & فرانسه & SF085 & qu & IFVC & صربستان & $803-1$ & س \\
\hline- & - & SF092 & 94 & ENSAT & فرانسه & $1009370-1(100 \mathrm{~K})$ & fis \\
\hline SPII & ايران & HC91 & 90 & Caussade semences & فرانسه & CSWW2X & is \\
\hline SPII & ايران & $10-59$ & 99 & ENSAT & فرانسه & $1009370-3(100 \mathrm{~K})$ & q \\
\hline- & - & H-100A-90RL8 & qท & ASGROW & فرانسه & $\mathrm{H} 158 \mathrm{~A} / \mathrm{H} 543 \mathrm{R}$ & iv \\
\hline ENSAT & فرانسه & SF109 & 91 & ASGROW & فرانسه & $\mathrm{H} 100 \mathrm{~A}$ & is \\
\hline ENSAT & فرانسه & SF105 & 99 & ASGROW & فرانسه & 15031 & 19 \\
\hline- & - & SF-023 & $1 .$. & ASGROW & فرانسه & H205A/83HR4 & D. \\
\hline
\end{tabular}

بين محيطها و لاينها در سطح احتمال يك درصد اختاف اختاف

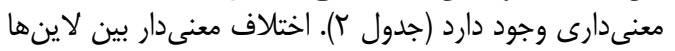

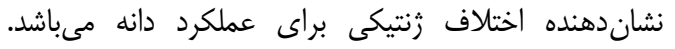
همجِنين جدول تجزيه واريانس نشان عان داد برهمكنش

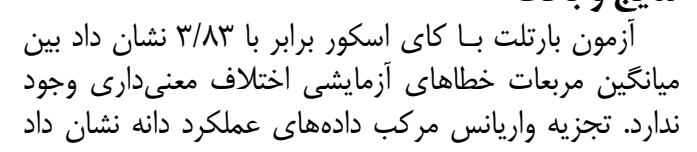


ارزيابى بإيدارى عملكرد لاينهاى اينبرد آفتابكردان در شرايط تنش شورى

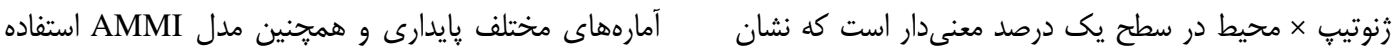

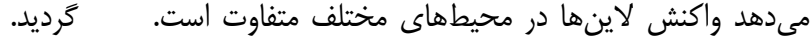

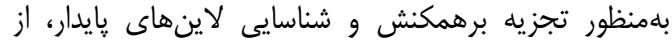

جدول r- تجزيه مركب واريانس بر اساس طرح بلوى كامل تصادفى براى عملكرد دانه در آفتابخردان روغنى Table 2. Combined analysis of variance based on RCBD for seed yield in oily sunflower

\begin{tabular}{|c|c|c|c|}
\hline ميانكين مربعات & مجموع مربعات & درجه آزادى & منابع تغييرات \\
\hline$\cdot / r^{* * *}$ &.$/ 99$ & $r$ & محيط \\
\hline.$/\left.\mu\right|^{* *}$ & $r / 4 \Lambda$ & $\wedge$ & تكرار داخل محيط \\
\hline$\cdot 1 \cdot \lambda^{* *}$ & $N / F$ & 99 & زَنوتي \\
\hline$\cdot / \cdot r^{* *}$ & $\Delta / V T$ & rqv & محيط × زنوتيب \\
\hline $.1 \cdot 1$ & $11 / V^{4}$ & v & اشتباه \\
\hline$q / \cdot r$ & & & ضريب تغييرات \\
\hline
\end{tabular}

توجه به اينكه فقط لاينهاى با كد الإن و وس عملكردى بيشتر

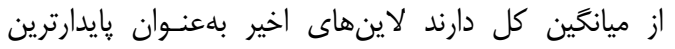

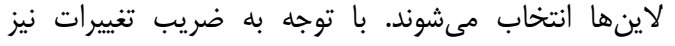

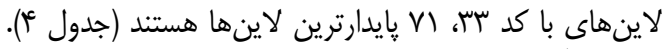

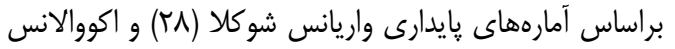

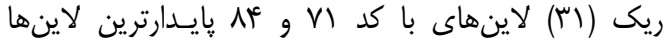

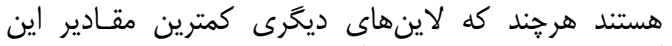

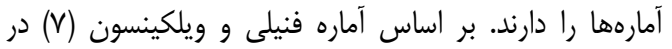

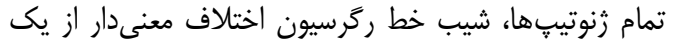

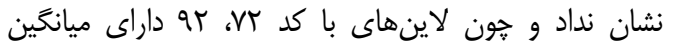

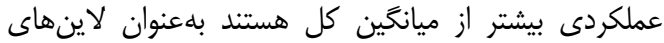

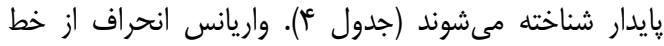

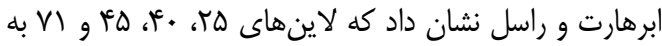

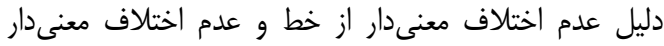

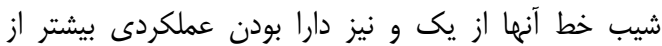

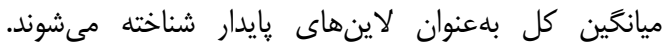

نتايج تجزيه يايدارى زنوتيبهاى مورد مطالعه بر اساس

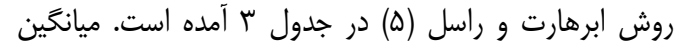

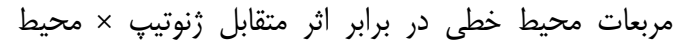

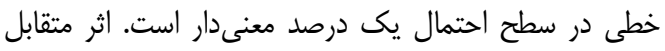

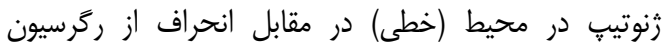

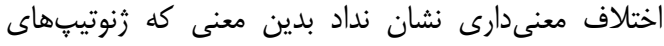

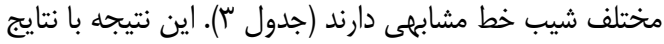

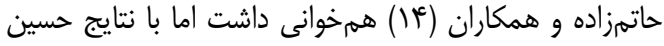

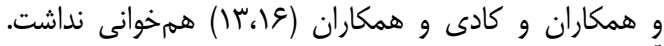

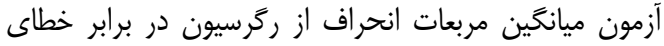
متوسط نشان داد كه در برخى زنوتيڤها

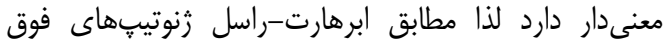

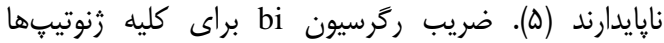

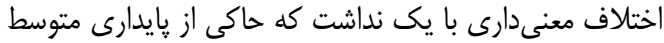

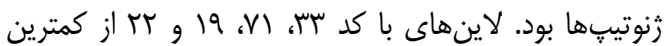

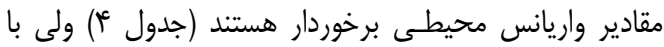

Table 3. Stability analysis based on Eberhart and Russell method for seed yield in oily sunflower

\begin{tabular}{|c|c|c|c|}
\hline ميانگَين مربعات & مجموع مربعات & درجه أزادى & منابع تغيير \\
\hline.$/ .14$ & $8 / 11$ & एव9 & كل \\
\hline 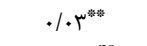 & r/qT & 99 & رنوتيب \\
\hline$\cdot \cdot \cdot v^{n^{n s}}$ & $T / M$ & $r .$. & محيط داخل زنوتيب \\
\hline$\cdot / \Lambda^{N=w s}$ & ru & 1 & محيط (خطى) \\
\hline$\cdot 1 \cdot 1^{\mathrm{ns}}$ & . & 99 & محيط (خطى) × زنوتيب \\
\hline $.1 \cdot 1^{\text {swat }}$ & r & $r .$. & انحراف از ركرسيون \\
\hline 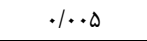 & $r / 91$ & var & خطاى متوسط \\
\hline
\end{tabular}

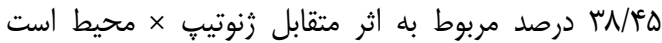

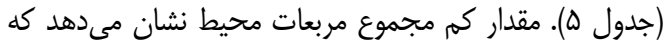

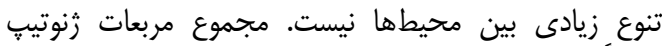

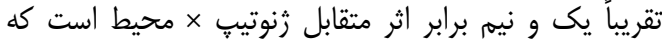

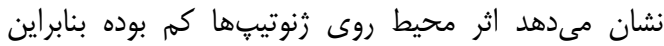

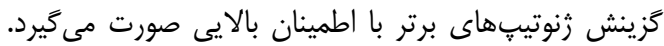

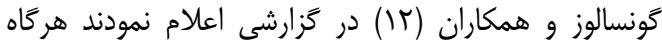

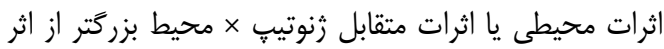

براساس شاخص سازگًارى هندسى (GAI) كه مبتنى بر

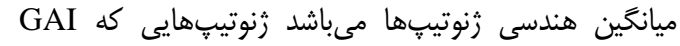

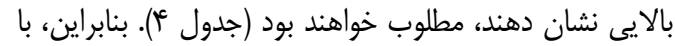

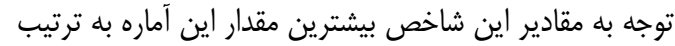

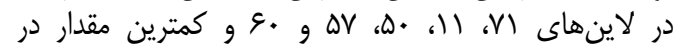

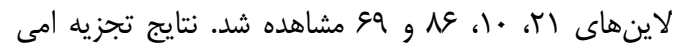

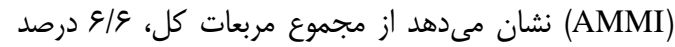

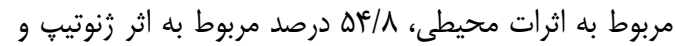


مفيدى براى تجزيه اثر متقابل زنوتيب × محيط بيان كردند

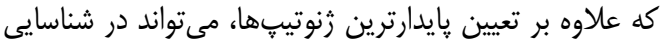

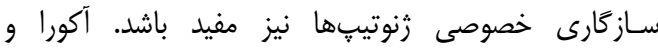

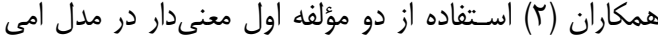

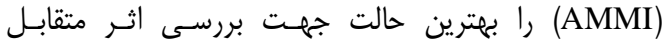

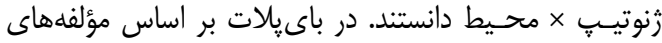

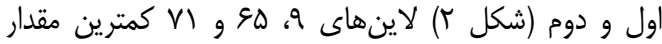

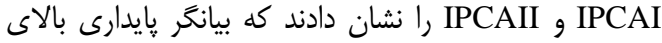

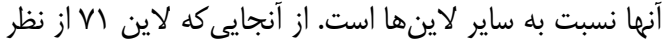

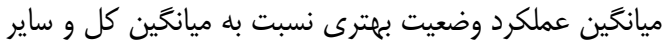

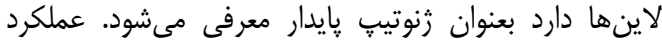

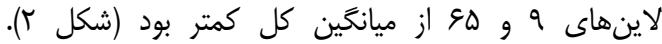

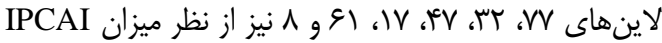

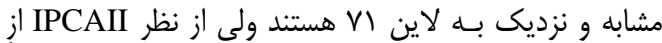

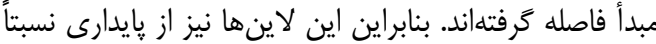

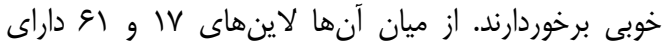

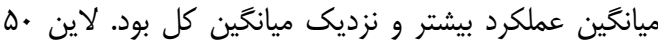

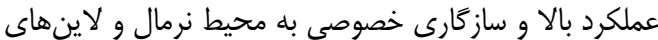

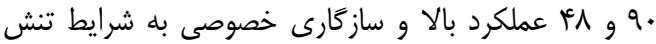

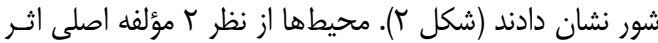

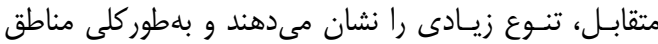

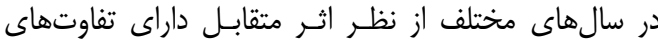

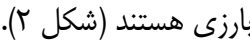

رنوتييى باشد كزينش زنوتيبهاى برتر تحت تأثير اثرات

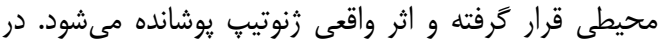

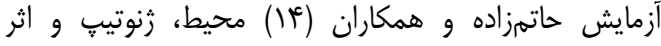

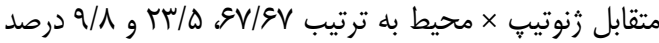

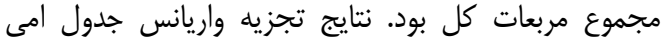

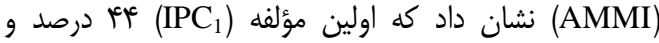
دومين مؤلفه (

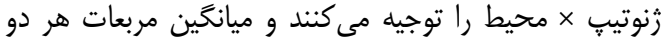

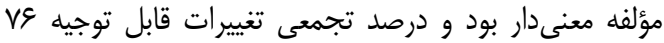

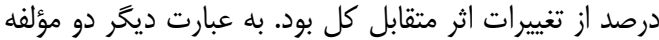

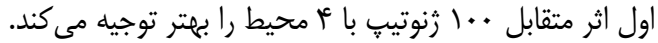

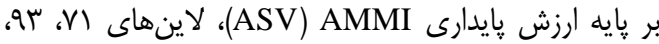

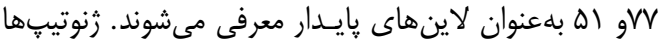

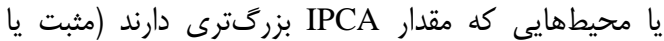

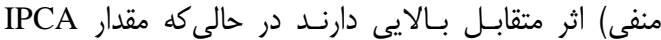

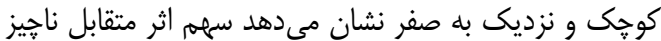

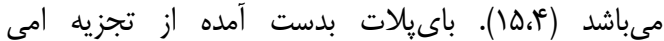

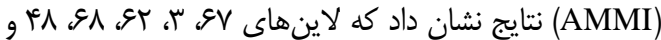

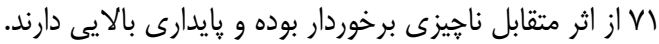

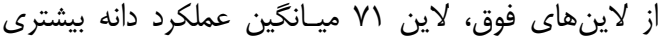

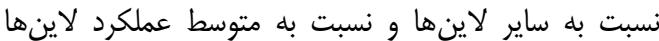

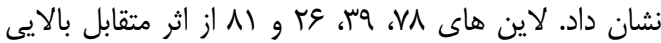

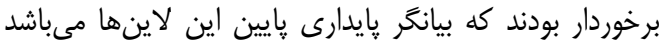

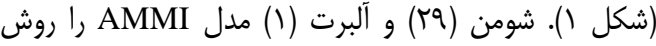

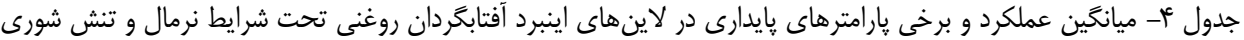
Table 4. Mean seed yield and some stability parameters in oily sunflower lines under salt and normal conditions

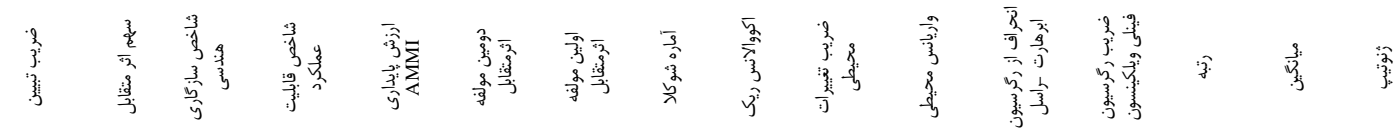

\begin{tabular}{|c|c|c|c|c|c|c|c|c|c|c|c|c|c|c|c|}
\hline $\mathrm{R}^{r}$ & GE\% & GAI & Ii & ASV & PCIr & PCI I & $\sigma \mathrm{i}^{r}$ & $\mathrm{~W}_{\mathrm{i}}^{r}$ & $\mathrm{cv}$ & $S_{x i}^{r}$ & $\mathrm{~S}_{\mathrm{di}}^{r_{1}}$ & $b_{i}$ & Rank & Mean & GEN \\
\hline f & . & $\mathrm{W} / \mathrm{.}$ & $\mid r / r q$ & $\cdot / T \cdot T$ &.$/ T$ & $\cdot / \cdot r$ &.$/ .1$ &.$/ . \mu$ & $\cdot / \cdot \Lambda$ &.$/ \cdot 1$ & $\cdot / \cdot 10^{n s}$ &. $\mid 9 \mathrm{~V}^{\mathrm{ns}}$ & $N$ & $1 N /\left.\right|^{6}$ & 1 \\
\hline$\Delta N / T$ & . & $\mid V / F$ & $18 / 19$ & .11 .1 & $-\cdot / 1$ &.$- / .1$ &.$/ .1$ &.$/ . r$ & $\cdot / \cdot 1$ &.$/ .1$ & $\cdot / \cdot \varphi^{n s}$ & $r / \Delta^{\mathrm{ns}}$ & 9. & $\mathrm{IV} / \mathrm{V}$ & r \\
\hline$V r / s$ &.$/$. & $\mathrm{rV} / \mathrm{q}$ & $1 \% / 9 v$ &.$|\cdot 1|$ & $-\cdot / \cdot 1$ &.$/ .1$ &.$/$. & $\cdot /$. &.$|+4|$ &.$/ . t r$ & $\cdot(\cdot)^{n s}$ & $1 / 9 Y^{n s}$ & $r$ & $r M / 1$ & $r$ \\
\hline$F \Delta / \Lambda$ & $\cdot / v$ & $r \cdot / V$ & $r I / A V$ &.$/ 4 \wedge 9$ &.$/ \cdot r$ &.$/ r$ & .1 .1 &.$/ . f$ & $\cdot 11 . r$ &.$/ .11$ & $\cdot / \cdot 1 \Delta^{\mathrm{ns}}$ & $r / .^{\text {ns }}$ & sr & $r I / F$ & f \\
\hline$r / \cdot$ &.$/ 1 \Lambda$ & $r r /$. & $r / / \kappa^{\prime}$ & ./Ifr & .1. & $-/ 1$ &.$/$. & .1 .1 & 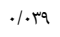 &.$/ . r$ & $\cdot / \cdot e^{\mathrm{ns}}$ & $-\cdot / r^{n s}$ & is & $r / r$ & $\Delta$ \\
\hline$r / r$ & . & $19 / 1$ & $\mid N / \notin \varepsilon$ & $\cdot \pi \cdot t$ &.$/ . r$ & $-/ / f$ &.$/ .1$ &.$/ . r$ &.$/ . \Delta 1$ &.$/ . t$ & $\cdot / \cdot \varphi^{n s}$ & $-\cdot / \mathrm{f}^{\mathrm{ns}}$ & ᄉ. & $19 / r$ & 9 \\
\hline$\Delta N / r$ &.$/ v$ & $r r / r$ & $|g / 9|$ & . TAT &.$/ 14$ & $-\cdot / \mathrm{V}$ &.$/ .1$ & .1 .4 & $.1 .9 \mathrm{~V}$ & $\cdot / \cdots 1$ & $\cdot / \cdot \Delta^{n s}$ & $-r / r^{\mathrm{ns}}$ & $\Delta r$ & $r r / s$ & $r$ \\
\hline $\mathrm{eV} / \mathrm{V}$ & $\cdot / M$ & $r r / G$ & $18 / 9 r$ & $\cdot / T M$ &.$/ 19$ &.$- / 10$ &.$/ . r$ & $\cdot / \cdot \Delta$ & $\cdot|\cdot 1|$ &.$/ .14$ & $\cdot / \cdot q^{n s}$ & $-r / \Delta^{n s}$ & ev & $r / r$ & $\wedge$ \\
\hline 1.18 &.$/$. & $W / 1$ & W/VE & .1 .14 &.$/$. &. .1 .1 &.$/$. & $\cdot /$. & . I. Tr &.$/ . .1$ & $\cdot(\cdot)^{n s}$ & $\cdot / \pi r^{n s}$ & $\wedge V$ & $|N /|$ & 9 \\
\hline$\Delta / \Delta$ &.$/ 1$ & $18 / 1$ & $r \Psi / N F$ & - /ISt & -.1 .4 &.$- / 11$ &.$/$. & .1 .1 & $.1 .4 F$ &.$/ . r$ & $\cdot / . . e^{\mathrm{ns}}$ & $\cdot \cdot / \kappa^{n s}$ & 99 & $19 / 9$ & 1. \\
\hline$r V / A$ &.$/$. & $\mu \epsilon / \Delta$ & $18 / 19$ & .1 .90 & -.1 .4 & -.1 .9 &.$/$. & $\cdot /$. &.$/ . T V$ &.$/ . r r$ & $\cdot / \cdot r^{n s}$ & $\cdot / N^{n s}$ & $r$ & $r \varphi / s$ & 11 \\
\hline$\Gamma \Delta / \Delta$ &.$/ 1$ & $r T / \Delta$ & $\mid \Delta / \Psi^{\prime}$ & .1 .94 &. .1 .9 & -.1 .0 &.$/$. & .1 .1 & .1 .48 &.$/ . t$ & $\cdot / \cdot r^{n s}$ & $1 / r^{n s}$ & DI & $r T / V$ & ir \\
\hline$N / 9$ &.$/ \% \Delta$ & $r / g$ & $r . / T \Delta$ & ./NF & rו/.-. & -.1 .4 & .1 .1 &.$/ . r$ &.$|+\Delta|$ &.$/ . .9$ & $\cdot / \cdots 1$ & $\cdot / v^{n s}$ & $v$ & $r r /$. & r \\
\hline $\mathrm{rV} / \mathrm{A}$ & $\cdot / v$ & $r q / r$ & $r V / \Delta T$ &.$/ \mu$. &.$/ 10$ &.$/ M$ &.$/ .1$ &.$/ . f^{f}$ & .1 .91 &.$/ .11$ &.$/ 19 \%$ & $r / r .{ }^{n s}$ & ir & $r \cdot / r$ & if \\
\hline$r / \Lambda$ &.$/$. & $r g / r$ & $r r / G \Lambda$ & .1 .91 & $-. .1 . r$ & -.1 .9 &.$/$. & $\cdot /$. &.$|\cdot r|$ &.$/ . .1$ & $\cdot(\cdot \cdot)^{n s}$ & $\cdot \pi^{n s}$ & TV & $r \varepsilon / r$ & 10 \\
\hline $9 / 9$ &.$/ \% \Delta$ & $r . / 9$ & $r N / N$ &.$/ 190$ & זו/. & $\cdot / \cdot v$ & .1 .1 &.$/ . r$ & .1 .09 &.$/ . .9$ & $\cdot / \cdot q^{n s}$ & $.199^{\mathrm{ns}}$ & $8 \Lambda$ & $r M / l$ & 19 \\
\hline$s \mathrm{~V} / \mathrm{G}$ &.$/ 1 \Lambda$ & $r \Delta / V$ & $r e / r q$ & . $/$ TS &..- .4 &.- .1 .1 &.$/$. & .1 .1 &.$/ . \Delta T$ & $\cdot / \cdot \Delta$ & $\cdot / . \cdot r^{\mathrm{ns}}$ & $1 / 99^{\text {ns }}$ & r. & $r q /$. & IV \\
\hline$g r / 1$ &.$/ M$ & $T V / T$ & $r q / \cdot V$ &.$/ I V^{e}$ &.$/ \cdot r$ &.$/ \pi$ &.$/$. &.$/ .1$ & $1.9 \pi$ & $\cdot / \cdots 1$ & $\cdot / \cdot \Delta^{n s}$ & $r / M \mathrm{~F}^{\mathrm{ns}}$ & rf & rV/A & 11 \\
\hline$\Delta 1 /$. &.$/ 1 \Lambda$ & $r r / q$ & $r r / F i$ & $\cdot 11 \cdot 0$ & .1 .9 & -1.9 &.$/$. & .1 .1 & r & $\cdot / \ldots$ & ${ }^{\prime} / *^{\mathrm{ns}}$ &.$- / 4 r$ & 19 & $r r / q$ & 19 \\
\hline $1 / 9$ &.$/ M$ & $r M / 1$ & $r . / 99$ & ./1rq & $-\cdot / \cdot 1$ &.$- / \cdot v$ &.$/$. &.$/ .1$ & $.1 .+\mathrm{F}$ &.$/ .+4$ & $\cdot / \cdot \varphi^{n s}$ & $\cdot \pi n^{n s}$ & so & $r \mid / r$ & r. \\
\hline$r q / 1$ &.$/ 1$ & $18 / 9$ & $r \Psi / \cdot \Delta$ & .11 .9 & $-* /$ & /. r &.$/$. & .1 .1 & .1 .90 &.$/ . .9$ & $\cdot / \cdot \varphi^{n s}$ & $1 / \mathscr{s} \Gamma^{n s}$ & १४ & $|v /|$ & rI \\
\hline $1 / 4$ &.$/$. & $r \cdot / r$ & $r g / r)$ & $\cdot / \cdot v r$ &.- .1 .1 &.$- / . \Delta$ &.$/$. & $\cdot / \cdot$ &.$/ .19$ & $\cdot /$. & $\cdot(\cdot \cdot)^{n s}$ & $-\cdot / 1^{n s}$ & $v_{\Delta}$ & $r \cdot / r$ & rr \\
\hline$\Delta / q$ & $\cdot / r \Delta$ & $1 / / 9$ & $r \Psi / \cdot r$ & THT & -.1 .4 &.$- / 19$ &.$/ .1$ &.$/ . r$ & .1 .48 &.$/ . r$ & $\cdot / \cdot \Delta^{n s}$ & $-\cdot / 0^{\mathrm{ns}}$ & 11 & $19 / 1$ & זr \\
\hline
\end{tabular}




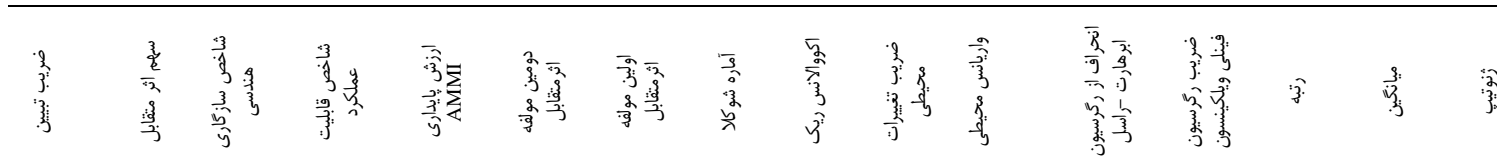

\begin{tabular}{|c|c|c|c|c|c|c|c|c|c|c|c|c|c|c|c|}
\hline $\mathrm{R}^{r}$ & GE\% & GAI & Ii & ASV & PClr & PCI & $\sigma \mathrm{i}^{r}$ & $\mathrm{~W}_{\mathrm{i}} \mathrm{r}^{-}$ & $\mathrm{cv}$ & $\mathrm{S}_{\mathrm{xi}}^{\mathrm{x}^{\prime}}$ & $S^{r_{d i i}}$ & $b_{i}$ & Rank & Mean & GEN \\
\hline Fq/1 & $\overline{T / R T}$ & $19 / 9$ & $T r / 1 \Delta$ & . MAF &.$- / T S$ &.$/ . \mu$ & $\%$ & $\%$ & "זואו & $1 . \% 9$ & 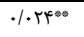 & $r / \Lambda r^{n s}$ & vi & $r \cdot / \Lambda$ & $r \mu$ \\
\hline १N/. &.$/ 1 \wedge$ & $T H / F$ & $T / / 19$ & TFF & -.1 .9 & .1 .9 & $\%$ & .1 .1 & .1 .99 & $/ \cdots \wedge$ &.$/$. ns & $r / q$ & $r_{F}$ & $r F / \Lambda$ & ro \\
\hline$r q / 1$ & $1 / .0$ & $T N$. & INFY & . $/ F F q$ & .11 & r & .1 .9 &.$/ r$ & .11 .9 & $.1 . r F$ & $1 \cdot \Gamma \Delta^{\omega 0}$ & $r / v r^{n s}$ & iv & $r a / r$ & rq \\
\hline$k / 8$ & . & $19 / 9$ & $19 / . \Delta$ &.$/ M$ &.$- / \cdot \Lambda$ & -.1 .9 & $\%$ & .1 .1 & .1 .94 & $\% v$ & $\%$...q ns & $\cdot / \Delta \mathrm{Vs}$ & ve & $19 / 9$ & $T r$ \\
\hline$N / 9$ & 年 & $\mid V / \Delta$ & $r / 11$ & $\cdot / \cdot \wedge \Delta$ & $.1 \cdot 1$ &.$- / . r$ & $\%$ &.$/ 11$ & $1.9 r$ & $1 . .9$ & $\quad / . \wedge^{n s}$ & $\cdot / v \Delta$ ns & 9) & $\mid V / V$ & $r \Lambda$ \\
\hline$r / \Lambda$ & . $/ r \Delta \Delta$ & $19 / 8$ & $19 / \%$. & . & 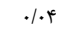 & $\cdot / \cdot \Lambda$ & $\%$ &.$/ 1$ &.$/ \Delta \Lambda$ &.$/ .99$ & $\% \cdots \wedge$ ns & $\mid(q)^{n s}$ & v & $19 / 1$ & rq \\
\hline$r r / 1$ & . $/ \pi \sim \Delta$ & $r \%$ & $T / \Delta A$ & ./NGF & $\cdot /$ & .1 .9 &.$/ . r$ &.$/ 1$ & $.1 .9 \mathrm{~V}$ & $\cdots 1$ & $\% \cdot \cdot v^{n s}$ & 1/Aه $\mathrm{ns}$ & $v^{e}$ & $r . / r$ & $r$. \\
\hline$\Delta S / F$ & $\cdot / 11$ & $N / N$ & $r V / \Delta$ &.$/ 119$ &.$- / . r$ &.$/ \cdot \Lambda$ &.$/ 1$ & $\%$ & $\% \cdot \Delta f$ & 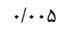 & /... ns & 1//99 ns & $\Delta f$ & $1 \Delta / \Delta$ & r \\
\hline$V V / \Delta$ & $\%$ & $\mid V / F$ & $r V / T F$ & $\cdot|\cdot v|$ &.$- / \cdot v$ &.$/ 1$ & $\%$ & $\%$ & 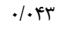 & 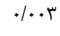 & $\cdot / \cdot 1^{\mathrm{ns}}$ & $1 / \Delta \Delta \Delta^{n s}$ & 94 & $\mid V / \Delta$ & rr \\
\hline $9 F / r$ &.$/ / \Lambda$ & $r / v$ & $T V / \Delta \Lambda$ &.$/ M V$ & .1 .9 & $-.1 . v$ & .1 .1 & $\%$ & $\%$ &. & $\% \ldots$ ns &.$- / \mathrm{Fns}^{\mathrm{ns}}$ & Fr & $r / / r$ & r \\
\hline $9 \pi / 8$ & $\%$ & $r / \Lambda$ & $r \cdot / N F$ & $.1 . \Delta q$ &.$- / .4$ &.$/ . r$ & $\%$ & $\%$ & $\% * r$ & $/ . . \mu$ &.$/$. ns & $1 / 1 / q^{n s}$ & 9. & $r / q$ & $\mu_{r}$ \\
\hline $19 / 1$ & $\%$ & $r)$ & $\mid v / / k q$ & /.Ar &.$/ 4$ &.$- / . \Delta$ & $\%$ & $\%$ & $/ \% \Delta$ & $\cdot \mid . .1$ & $\cdot(\cdot .)^{\text {ns }}$ & $\cdot F \Lambda^{n s}$ & 99 & $r / 1$ & ro \\
\hline $11 / 9$ &.$/ 1 \wedge$ & NTr & $r T / \Delta F$ &.$/ . \Delta \wedge$ & $\%$ &.$/ . f^{e}$ &.$/ 1$ & $\%$ & $\%$. Df & 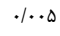 & $\cdot(\cdot .)^{\mathrm{ns}}$ & $r / \% \mathrm{rs}$ & $\Lambda \Delta$ & $W_{F}$ & rq \\
\hline$\Delta \Delta / \bar{\varphi}$ & $\cdot / M \Lambda$ & $\mathrm{r} / \mathrm{I}$ & r/aT & /.人 & .1 .9 & $.1+\kappa$ &.$/ 1$ & $\%$ & $\cdot|+4|$ &.$/ . F F$ & $\cdot \cdot \cdot r^{\mathrm{ns}}$ & V/AT & $\wedge$ & $r / / r$ & $r v$ \\
\hline$\mu / r$ &.$/ \pi \Delta$ & $r \cdot / F$ & Tr/TS & ./1Es &.$- / . r$ &.$- / 1$ & $\%$ &.$/ 1$ & $\% \leftarrow$. & . & $\cdots, \cdot r_{n s}$ & $-1 / /^{n s}$ & Vr & $r \cdot / \Delta$ & rᄉ \\
\hline $10 / 9$ & $1 / \Delta A$ & $T \psi \%$ & 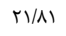 & $\cdot / 4 \cdot 1$ & .1 .9 & $\cdot / \pi \Lambda$ & 1.99 & 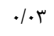 & ת M & 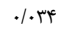 & 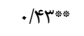 & $r / 4 r^{n s}$ & ו & $r \Delta / /$ & rq \\
\hline १९/8 & $\cdot / / \Lambda$ & $T V / V$ & $1 \mathrm{~V} / \mathrm{Fq}^{\prime}$ & . & $\%$ & .1 .9 & .1 .1 & $\%$ &.$/ \Delta F$ & $.1 . .9$ & /.. ns & $r / \Delta r^{n s}$ & r & TN. & r. \\
\hline$v^{*} / F^{-}$ & $\cdot / v$. & $T+/ \mu$ & TD/qT & $\cdot / / V$ & .1 .9 &.$- / 1$ & $\%$ & .1 .1 & .1 .91 & 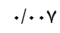 & $\cdot \cdot . r^{\mathrm{ns}}$ & $-r / /^{\mathrm{ns}}$ & rq & $r F / 8$ & i) \\
\hline $9 N 1$ & $\cdot / \cdot$ & $r) /$. & $r / / R \mu$ & /.Kr &.$/ . r$ &.$- / . r$ & $\%$ & $\%$ &.$/ 11$ & $\cdot / . .1$ & $\% \ldots$ ns &. $\mid q 4$ ns & v. & $r)$ & rr \\
\hline$r \cdot / \Delta$ & . & $r \cdot / V$ & $r / / r r$ &.$/ T \Delta$ & $.1 . \Delta$ & $\cdot / I V$ & 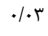 &.$/ 1$ &.$/ . \wedge 9$ & זו./. & $\cdot / 11)^{\mathrm{ns}}$ & $r / F^{\text {ns }}$ & $9 v$ & $r / r$ & $r \mu$ \\
\hline$r / r$ & $\cdot \mid M$ & $r q / v$ & $r F / \% A$ &.$/ r r$ &.$- / \pi r$ & -0.04 & $.0 \Delta$ & $\%$ & .1 .19 &.$/ . \mathrm{V}$ & $1.5 \Delta^{* 0}$ & $\cdot / \Lambda^{n s}$ & 1. & $r .18$ & HF \\
\hline १९/६ & $\%$ & $T H / F$ & INTY & $.1 . v f^{f}$ & -.1 .9 & $\%$ & $\%$ & $\%$ & $\% * \wedge$ &...$p$ & $\% \ldots$ ns & $r / r^{\mathrm{ns}}$ & rv & $r \xi / 8$ & is \\
\hline $99 / 1$ &.$/ 1 \wedge$ & $r N / r$ & $\mathrm{r} / / \mathrm{AF}$ & . &.$- / .4$ & $\% \wedge$ &.$/ 1$ & $\%$ &.$/ . \Delta 1$ & $\cdots \infty$ & $\% \ldots$ ns & $r / F r$ & 19 & $r N V$ & 19 \\
\hline$F N / F$ & $\%$ & $r \% /$. & $r N / 9$ & 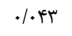 &.$/ 4$ &.$/ 1$ & $\%$ & $\%$ & . ז &.$/ . .1$ & $+/.)^{\text {ns }}$ & $\cdot / \mathrm{A}^{\mathrm{ns}}$ & $\Delta V$ & $r / 1$ & er \\
\hline$F / V$ & . $/ r \Delta \Delta$ & $r \cdot / \Delta$ & $r r / \kappa \Lambda$ & וTו/. & זו/. &.$- / .1$ & $\%$ &.$/ 1$ & . $1 . \mu q$ & /..r & $\mid \cdot . \Delta \Delta^{n s}$ & $-\cdot / k^{n s}$ & 9 & $r \cdot / \Lambda$ & rᄉ \\
\hline$s / 4$ & $1 / . \Delta$ & $r V / r$ & $\mathrm{r} / \mathrm{vq}$ & $\cdot \pi \cdot F^{2}$ & $\% / 1$ &.$- / 1 \pi$ & .1 .9 & $\%$ & .1 .91 & /.r. & $1 / \% \Lambda^{* 00}$ & $1 / r^{\mathrm{ns}}$ & $r$ & TNM & 19 \\
\hline $9 T / \Lambda$ & $\cdot / v$ & $r F / \Delta$ & TNAI & $\%$ &.$- / . t$ &.$/ I V$ & $\%$ &.$/ 1$ & $.1 .9 \mathrm{~V}$ & $.1 . \pi r$ & \% & $F / V^{n s}$ & r & rel. & $\Delta$. \\
\hline $\mathrm{FV} / \mathrm{r}$ & $\%$ & $r g / T$ & ra//4s & . $/ \mu r$ &.$- / . \mu$ & $\% . r$ & $\%$ & $\%$ & $\% \cdot r V$ &.$/ .1$ & $(\mid . .)^{\text {ns }}$ & $\cdot / \mathrm{A}^{\mathrm{ns}}$ & rM & TS/T & D) \\
\hline$s / V$ & . $/ \Delta r$ & $r / s$ & TS/GY &.$/ 191$ & $\% / 1$ & $\cdot / \pi r$ &.$/ 1$ & $\%$ & $\%$. vg &.$/ 1$. & $\pi / 1 \mathrm{Fns}^{\mathrm{ns}}$ & $\cdot / N \mathcal{n}^{\mathrm{ns}}$ & $\Delta S$ & $r T / 1$ & $\Delta r$ \\
\hline $\mid r / 1$ & $\cdot / v$ & $r / r$ & $r F / q T$ & $\cdot / T \Delta \mid$ &.$- / 10$ &.$- / 1 f$ &.$/ 1$ & 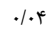 &.$/ 191$ & .1 .10 &.$/ 19 \%$ & $1 / r \wedge^{n s}$ & $\Delta \wedge$ & $r / / q$ & $\Delta r$ \\
\hline$r F / s$ &.$/ 1 \wedge$ & $I V / r$ & $r N \cdot V$ & $.1 \cdot 19$ & $\%$ & -.1 .9 & $\%$ &.$/ 1$ & $\% . \Delta q$ & 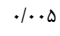 & $\cdot / \cdot \Delta^{\mathrm{ns}}$ & $1 / 4 \mathrm{HS}^{\mathrm{ns}}$ & 98 & $\mid V / F$ & $\Delta f$ \\
\hline$F^{*} / \Lambda$ & $\cdot / / \Lambda$ & $T \%$ & $r \cdot \Delta F$ & שr//. & $\%$ & .1 .9 & $\%$ &.$/ 1$ & $\% \Delta r$ & $\cdots \infty$ & $\mu_{0}$ ns & $1 / \Delta \Lambda^{n s}$ & r. & $T F / T$ & $\Delta \Delta$ \\
\hline 9.11 & . $/ \pi \Delta$ & $r \mu / \mu$ & $\mathrm{ra} / \mathrm{v}$. & / / V r &.$- / 1 f$ & $\% \cdot v$ &.$/ 1$ & $\%$ & $\%$. A & זו. & $\cdots \cdot \cdot r^{n s}$ & $r / \Delta q \mathrm{~ns}$ & Hr & $r r / q$ & $\Delta S$ \\
\hline $5 \% / \mu$ & $\cdot / / \Lambda$ & $r r / q$ & $r r / F r$ & $\cdot \pi \cdot 1$ & .1 .0 &.$/ 1 F$ & $\%$ &.$/ 1$ &.$/ \Delta S$ & $\% v$ & 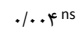 & $r / r r^{\text {ns }}$ & F & $r \% / F$ & $\Delta r$ \\
\hline $9 \mathrm{~V} / \mathrm{r}$ &.$/ 1 \wedge$ & $T N /$ & $r q / \% \Delta$ &.$/ \Delta r$ & $\%$ & $\%$ & $\%$ &.$/ 1$ & $1 / .+q$ & $\cdots \infty$ & $\% \cdot r^{\text {ns }}$ & $1 /\left.9\right|^{\mathrm{ns}}$ & M & rq/. & $\Delta \Lambda$ \\
\hline SN/9 & $\%$ & $r \Delta / 1$ & rNq. & $1 . \Delta 9$ & $.1 . F$ &.$\%$ & $\%$ & $\%$ &.$/ . \mu$ &..$\cdot r$ & $\cdot(\cdot .)^{\text {ns }}$ & $1 / 1 Q^{n s}$ & rr & $r \Delta / r$ & $\Delta q$ \\
\hline$r F / r$ & $\cdot / / \Lambda$ & $r r / q$ & $r . / 9 \Lambda$ &.$/ N Q T$ &.$- / .1$ & .1 .9 & $\%$ &.$/ 1$ &.$/ \Delta f$ & $\% v$ & 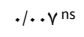 & $1 / \Delta q \mathrm{~ns}$ & $\Delta$ & $r \psi / F$ & 9. \\
\hline$r r / \Lambda$ &.$/ \pi \Delta$ & $T \psi /$ & r./vg & $\cdot 1 / .1$ &.$/$ &.$- / 11$ &.$/ 1$ & $\%$ & $.0 \Delta q$ & $\% v$ & $\cdot / \cdot \wedge^{\mathrm{ns}}$ & $|/ r|^{\mathrm{ns}}$ & rq & $r E / r$ & 9) \\
\hline $95 / 9$ &.$/ \% \Delta$ & $r 9 / 9$ & $F r / q \Delta$ &.$/ 191$ &.$- / 19$ & .1 .1 &.$/ 1$ &.$/ \%$ & $\% \mathrm{vV}$ &.$/ .1 r$ & $\cdot 1 . . \gamma^{n s}$ & $r / A \&$ ns & זq & $T V / r$ & 95 \\
\hline$T V / \Delta$ & $\cdot / M \Lambda$ & $r / \bar{\varphi}$ & $r F / / \gamma$ & . & .1 .9 &.$- / . V$ & $\%$ &.$/ 1$ & /. &.$/ . .1$ & $\cdot(\cdot .)^{\text {ns }}$ & $q^{n s}$ & if & $r \mu / 8$ & $9 \pi$ \\
\hline$g V / r$ & $\%$ & $19 / \Delta$ & rT/FA &.$/ . v g$ &.$- / \cdot v$ &.$- / . r$ & $\%$ & $\%$ & $.1 \% \Delta$ & ...r & $\cdots, \cdot r^{\text {ns }}$ & $1 / \Delta V^{n s}$ & $\mathrm{ru}$ & $19 / 8$ & 94 \\
\hline$Q N / \mu$ & $\cdot /$. & $\mathrm{IV} / \mathrm{V}$ & $r r / . V$ &.$/ .11$ &.$- / .1$ &.$/ 1$ & $\%$ & $\%$ & 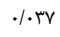 &.$/ . r$ & /.. ns & $1 / 49 \mathrm{~ns}$ & 19 & $\mathrm{IV} / \mathrm{A}$ & $9 \Delta$ \\
\hline $18 / 4$ & $\cdot / M \Lambda$ & $\mathrm{IV} / \mathrm{V}$ & $r \cdot / \mu$. &.$/ . v g$ &.$/ . \Delta$ & -.1 .4 & $\%$ &.$/ 1$ & \% & . & $\| k^{n s}$ & $\cdot / v Y^{n s}$ & $M$ & $\mid V / A$ & 99 \\
\hline $19 / 0$ & $\cdot / \cdot$ & $r N / \mu$ & $\mathrm{rV} / \mathrm{Tr}$ & /.r. & $\% . r$ & $\%$ & $\%$ & $\%$ & $\cdot / \cdot r$ &.$/ . .1$ & $\cdot /\left.\cdot\right|^{\mathrm{ns}}$ & $\cdot / 4 \cdot \mathrm{ns}$ & r. & $r N F$ & $9 V$ \\
\hline $95 /$. & $\cdot /$. & $r \cdot / r$ & $r V / \cdot r$ & .1 .99 &.$/ . \infty$ & $\%$ & $\%$ & $\%$ & $\%$ & . & $\cdots \cdot . r^{\mathrm{ns}}$ & $1 / 4 \mathrm{HS}^{\mathrm{ns}}$ & ir & $r \cdot / F$ & 91 \\
\hline$N / r$ & . /NA & $19 / 9$ & $r r / . r$ & $\cdot / \cdot v r$ & $\%$ & $.1 . \Delta$ & $\%$ &.$/ 1$ &.$/ . \Delta 1$ & $\cdot / . .4$ & $\%$... ns & $\cdot / \Delta 9 \mathrm{~ns}$ & १ง & $\mid v /$. & 99 \\
\hline $\mid * \%$ & . $/ \Delta r$ & rq/. & $r r / \Lambda \Delta$ & . /NA & -.1 .9 &.$- / T$ &.$/ 1$ & $\%$ &.$/ \Delta s$ & $\% v$ & $\% \ldots q^{\text {ns }}$ & $-1 / \cdot \mathrm{ns}$ & 19 & $\mathrm{rq} / \mathrm{s}$ & v. \\
\hline$V Y / 8$ & $\cdot /$. & r\$1 & $r .1 .1$ &.$/ .1 f$ & $\%$ &.$- / .1$ & $\%$ & $\%$ &.$/ 1$ & $\bullet \ldots$ & $\% \ldots$ ns & $\cdot / \mu r^{n s}$ & 1 & 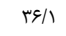 & n \\
\hline$S / V$ & $\cdot / M$ & $r / f$ & $r V / S Y$ & $\cdot \pi \cdot q$ & .119 & .1 .9 & $\% r$ & $.0 \Delta$ &.$/ A V$ & r./. & $.1 .19 \%$ & $-1 / \mathrm{ns}$ & $\Delta q$ & $r / 9$ & $r r$ \\
\hline$r \Delta / \Lambda$ & $\cdot / v$. & $r \Delta / V$ & $T F / F r$ & $\cdot / / V$. & -.1 .9 &.$- / 11$ &.$/ 1$ & $\%$ & .1 .90 & $\% \wedge$ & $\% \ldots q^{\text {ns }}$ & $-1 / \Delta^{n s}$ & rq & $r 91$ & $v^{r}$ \\
\hline $\mathrm{FV} / \mathrm{F}$ & 䧑 & $r e / F$ & $\mid \psi / \cdot \Lambda$ & $\cdot / 1 \Lambda$ & $-\cdot / / V$ &.$/ \%$ &.$/ 1$ & $\%$ & 1.9. & .1 .19 & $\cdot / 1 r^{\mathrm{ns}}$ & $r / A r^{n s}$ & $r$ & $r \Delta / r$ & $v^{e}$ \\
\hline$r \cdot / \mu$ & $\%$ & $\mid W / S$ & $T H / N \Delta$ & $\% \cdot \Delta T$ &.$- / . \Delta$ & .1 .1 & $\%$ & $\%$ & $\%$ & $. \cdot . r r$ & $\cdots \cdot . r^{\text {ns }}$ & . $/ v$ ns $^{\prime}$ & qr & $\mid W / s$ & va \\
\hline$\Lambda r / r$ & $\%$ & $r \mu / \mu$ & $\mathrm{T} / \mathrm{AS}$ & .1 .91 & $\% \cdot r$ & $\%+F$ & $\%$ & $\%$ & $\% . \mu F$ & $1 / . . F$ & $\cdot / \cdot 1^{\text {ns }}$ & $1 / A r^{n s}$ & is & $r \% / \Delta$ & ve \\
\hline $98 / 9$ & 年 & $r . / q$ & $r q / r q$ & $.1 . f^{e}$ &.$- / .4$ & $\%$ & .1 .1 &.$/ \%$ &.$/ \cdot v 1$ & $1 / .99$ & 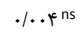 & $r / \Delta)^{n s}$ & 99 & $r / r$ & w \\
\hline$r r / 1$ & 1/qu & $r V / r$ & $r / / v \Lambda$ &.$/ F v g$ &.$- / . r$ & 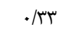 & $\%$ & $\cdot 11$ & שאואי & . & 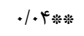 & $r / M^{n s}$ & 10 & $\mathrm{r} / \mathrm{s}$ & $\mathrm{vA}$ \\
\hline$r r / s$ & . & $r / q$ & $r r / q \Delta$ &.$/ \Delta M$ & -.1 .0 & -11. &.$/ 1$ & $\%$ & .1 .99 & $\cdots \wedge$ & $1 / . q^{\text {ns }}$ & $-1 / \mathbb{F}^{\mathrm{ns}}$ & $\Delta f$ & $r \pi / r$ & va \\
\hline
\end{tabular}


ادامه جدول

\begin{tabular}{|c|c|c|c|c|c|c|c|c|c|c|c|c|c|c|c|}
\hline $\begin{array}{l}2 \\
3 \\
3 \\
3 \\
3\end{array}$ & $\begin{array}{l}\frac{3}{2} \\
\frac{2}{2} \\
\frac{3}{3} \\
\frac{3}{3}\end{array}$ & 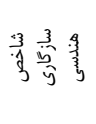 & 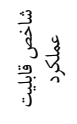 & $\overline{\frac{3}{3}}=$ & 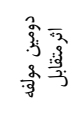 & 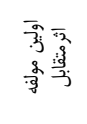 & 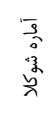 & $\begin{array}{l}\overline{5} \\
\frac{2}{7} \\
3 \\
\overline{3} \\
\overline{3}\end{array}$ & $\begin{array}{l}2 \\
3: \\
3: \\
\vdots 3 \\
\frac{3}{3}\end{array}$ & 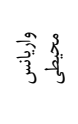 & 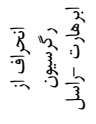 & 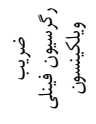 & 3 & $3_{3 .}^{3}$ & $\begin{array}{l}3 \\
3 \\
3: \\
3\end{array}$ \\
\hline $\mathrm{R}^{r}$ & $\mathrm{GE} \%$ & GAI & Ii & ASV & PCIr & PCII & $\sigma \mathrm{i}^{r}$ & $\mathrm{~W}_{\mathrm{i}^{r}}$ & $\mathrm{cv}$ & $S^{r} r_{i}$ & $\mathrm{~S}_{\mathrm{di}}^{r_{\mathrm{di}}}$ & $b_{i}$ & Rank & Mean & GEN \\
\hline $9 N / 4$ &.$/ 1 \Lambda$ & $W / V$ & $19 / \Delta \Lambda$ &.$/ . \wedge r$ & -.1 .9 & .1 .4 & $\%$ &.$/ .1$ & .1 .09 & $.1 . .9$ & $\%{ }^{\mathrm{ns}}$ & $r / 4 f^{n s}$ & N & $1 N 9$ & ᄉ. \\
\hline$r / \Delta$ & $1 / 4$ & $r v / \cdot$ & $r q / \kappa \Delta$ & $\cdot / r V \Delta$ & $\cdot \pi$. & $-\cdot / r r$ &. & $\cdot 1 \cdot 1$ & $\cdot 11 \cdot 1$ &.$|\cdot r|$ & $\cdot / \cdot \Gamma \mid *$ & $-r / g^{n s}$ & 91 & $r / 9$ & 11 \\
\hline $1 / 9$ & $\cdot / 1 \Lambda$ & $1 / / 9$ & $r q / 9 q$ &.$/ \mathrm{V}$. & .1 .9 &.$- / 11$ & $\%$ & .1 .1 & $.1+41$ &.$/ . r$ &.$/ .+f^{n s}$ & $-\cdot / r^{\mathrm{ns}}$ & NT & $19 /$. & NT \\
\hline$<q /$. & $\%$ & $r E / \tau$ & $r T / 1 Q$ &.$/ . \wedge t$ &.$- / \cdot v$ & $\%$ & $\%$ &.$/$. & צץ.|. & . . . & $\cdot / . r^{\mathrm{ns}}$ & $1 / / \varepsilon^{\mathrm{ns}}$ & ro & $r \kappa / \Delta$ & $\Delta r$ \\
\hline$r \cdot / r$ & $\cdot / \cdot$ & $r 1 / r$ & $r N \wedge \Delta$ & .11 .9 & -.1 .4 & $-\cdot / \cdot v$ & $\%$ & $*$. & I. & /..r & $\cdot / \cdot r^{n s}$ & $\cdot / N \mathbb{F}^{n s}$ & st & $r M / r$ & NF \\
\hline ry/g & $\cdot / 1 \Lambda$ & $r F / \Delta$ & $r \cdot / r r$ &.$/ 109$ &.$- / .1$ &.$- / 11$ & $\%$ & .1 .1 & r. &.$/ . r$ & $\cdot . . r^{n s}$ &.$- / 9 \mathrm{~ns}$ & ro & $r F / \varepsilon$ & $\Lambda \Delta$ \\
\hline$V \pi / T$ & $\cdot / v$ & $18 / 1$ & $r \cdot / \% q$ & / IVT &.$/ 1 f$ & $-\cdot / \cdot v$ & .1 .1 & .1 .4 & .1 .99 & $\cdot / \cdot v$ & $\mid \cdot . \mu \mathrm{rs}$ & $-r / \Psi^{\mathrm{ns}}$ & $1 .$. & $\mid g / F$ & $1 \varepsilon$ \\
\hline$F+/ 1$ & $* \cdot$ & $r r / q$ & $r q / \Delta \varphi$ & $\cdot / \cdot v r$ &.$- / .1$ &.$/ . \Delta$ & $\%$ & 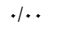 & Tr & $\| \cdot \cdot r$ & $\| \cdot . r^{n s}$ &.$/ 9 y^{\mathrm{ns}}$ & fi & $r r / q$ & $\Lambda V$ \\
\hline$r . /$. & $\cdot / 1 \Lambda$ & $I V / \Delta$ & $r \cdot / \Lambda$. &.$/ 19$ & .1 .1 &.$- / \cdot 1$ & $\%$ & .1 .1 & $\% \cdot \Delta F$ & $\cdot / \cdot \Delta$ &.. .9 ns & $1 / \cdot 1^{\mathrm{ns}}$ & qז & $\mid V / S$ & $M$ \\
\hline$V / r$ & $\cdot / 1 \Lambda$ & $\mathrm{IV} / \mathrm{r}$ & $r F / g r$ & $\cdot / .$. &.$/ .0$ &.- .1 .9 & $\%$ &.$/ .1$ &.$|+4|$ &.$/ . r$ & $\| / . .4 n s$ & $\cdot / \propto \Delta^{\mathrm{ns}}$ & 90 & $\mid V / F$ & 19 \\
\hline$N / 9$ & $\cdot / r \Delta$ & $r Y / G$ & $r M / A f$ &.$/ 199$ &.$/ 1 r$ &.$- \cdot 1 \cdot 1$ &.$/ .1$ &.$/ .4$ & . $/ \Delta \mu$ & $\| \cdot \Delta$ & $\cdot / \cdot v^{n s}$ & $-\cdot / v^{n s}$ & $\Delta$. & $r r / q$ & q. \\
\hline $1 \pi / \Lambda$ & $\cdot / 1 \Lambda$ & $r \cdot / r$ & $r q / r r$ & 快 & -.1 .9 &.$- / . \varphi$ & $\%$ & $.1 \cdot 1$ & $.1 .+\Delta$ & 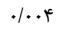 &...$q^{\mathrm{ns}}$ & $\cdot|\lambda|^{\mathrm{ns}}$ & 11 & $r \cdot / \Delta$ & 91 \\
\hline $\mid \nabla \Delta /$. & $\cdot / \cdot$ & $19 / r$ & TNFD & . Iro & .1 .9 & $\% \cdot v$ & $\%$ & $\cdot / \cdot$ &.$/ .4$. & $\cdot / \cdot r$ & $\| . . r^{n s}$ & $1 / 1 \mu^{n s}$ & va & $19 / r$ & ar \\
\hline$\varphi g / V$ & $\%$ & $r / / \Lambda$ & $r r / . r$ &.$/ . r$. & . & $\%$ & $\%$ & $\cdot / \cdot$ & r. &.$\cdot \cdot r$ & $\cdot / . \cdot r^{n s}$ & ./Q६ ns & st & $r M / \Lambda$ & q \\
\hline $19 / \pi$ &.$/ \% \Delta$ & $r / V$ & $r / q r$ & ./145 &.$- / . r$ &.$/ 1$. & $.1 \cdot 1$ &.$/ . r$ & $\cdot / \cdot \mathrm{V} \wedge$ &.$/ .14$ & $\| . . r^{n s}$ & $r / q V^{n s}$ & 9 & $r r / g$ & $9 f$ \\
\hline$\Lambda \Delta / \Lambda$ &.$/ \% \Delta$ & $r q / \Delta$ & $r r / 4$ & $\cdot / M M$ &.$- / N r$ &.$/ 1$ & .1 .1 &.$/ . r$ & $\cdot / \cdot v 1$ & $.1 \cdot 11$ & $\| . . r^{n s}$ & $r / 1 q^{n s}$ & if & $r \cdot / r$ & 90 \\
\hline$F \Psi / 4$ &.$/$. & $r r / r$ & $r F / q \Lambda$ & .1 .94 &.- .1 .9 &.$- / .0$ & $\%$ &.$/$. & . $1.4 q$ & /..r & $\cdot . \cdot r^{n s}$ & $1 / 1 \Delta^{\mathrm{ns}}$ & $\Delta \Gamma$ & $r r / F$ & 98 \\
\hline$\Lambda T / \cdot$ &.$/ \% \Delta$ & $T V / T$ & $F r / F q$ &.$/ 198$ & .1 .9 & $-\cdot / / r$ &.$/ \cdot 1$ &.$/ .4$ & . $1.4 T$ &...$t$ &.$/ .1^{\mathrm{ns}}$ & $-1 / \wedge^{\mathrm{ns}}$ & ro & $r V / F$ & १४ \\
\hline$\%$ & $\cdot / v$. & $r \mid / s$ & $r N \cdot G$ & $\cdot 11 \cdot 9$ & $-\cdot / 1$ & $-\cdot / \cdot r$ & .1 .1 & .1 .4 & $\cdot / \cdot \lambda f$ & . & $\cdot / \cdot 1 \wedge *$ & $\cdot / N F^{n s}$ & $\Delta \Delta$ & $T r / r$ & 91 \\
\hline $9 . / 4$ & $\cdot / \Gamma \Delta$ & $r r / q$ & 1 & $\cdot / r \cdot r$ & $\cdot / r$. & $\%$ &.$/ .1$ &.$/ . r$ & $\% \cdot v q$ &.$/ .1 T$ & $\cdot \cdot \cdot r^{n s}$ & $r / r V^{n s}$ & 18 & $r \mu / F$ & 99 \\
\hline $\mathrm{FV} /$. &.$/ M \Lambda$ & $r \cdot / r$ & WNAV & $\cdot / 1 \cdot 1$ & $-\cdot / 1$ &.$- / .1$ & $\%$ &.$/ .1$ & .1 .09 &.$/ .9$ & $\cdot / \cdot \Delta^{\mathrm{ns}}$ & $1 / N \mathbb{F}^{n s}$ & $v r$ & $\begin{array}{l}r \cdot / f \\
r V / r f\end{array}$ & $\begin{array}{c}1 . . \\
\text { ميانكين }\end{array}$ \\
\hline
\end{tabular}

جدول ه- تجزيه واريانس مركب و تجزيه امى (AMMI) اثر متقابل ثنوتيب × محيط براى عملكرد دانه در لاينهاى اينبرد آفتابخردان روغنى

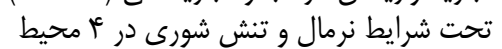

Table 5. Combined analysis of variance and AMMI analysis of GE interaction for grain yield in oily sunflower lines

\begin{tabular}{|c|c|c|c|c|}
\hline سهمم اثر متقابل & ميانگين مربعات & مجموع مربعات & درجه أزادى & منابع تغييرات \\
\hline &.$/ T^{\circ}$ & $r / \leftarrow \wedge$ & $\wedge$ & تكرار / محيط \\
\hline 8/99 & (4) &.$/ 99$ & r & محيط \\
\hline$\Delta F / \lambda \mid$ & $\cdot / \cdot \Lambda^{* \infty}$ & NIF & 99 & رثنتيب \\
\hline$r N F \Delta$ & $1 . r^{\circ}$ & $\Delta / v 1$ & rqv & محيط × زنوتيب \\
\hline $\mathrm{r} / / \Lambda$ & $.1 \cdot 1 \Lambda^{\circ}$ & I/VAT & 99 & مؤلفه دوم \\
\hline \multirow[t]{2}{*}{ r } &.$/ .1 f^{\mathrm{ns}}$ & 1/499 & qv & مؤلفه سوم \\
\hline & .1 .1 & $11 / v e$ & var & خطاى متوسط \\
\hline
\end{tabular}


$\wedge$

ارزيابى پايدارى عملكرد لاينهاى اينبرد آفتابخردان در شرايط تنش شورى

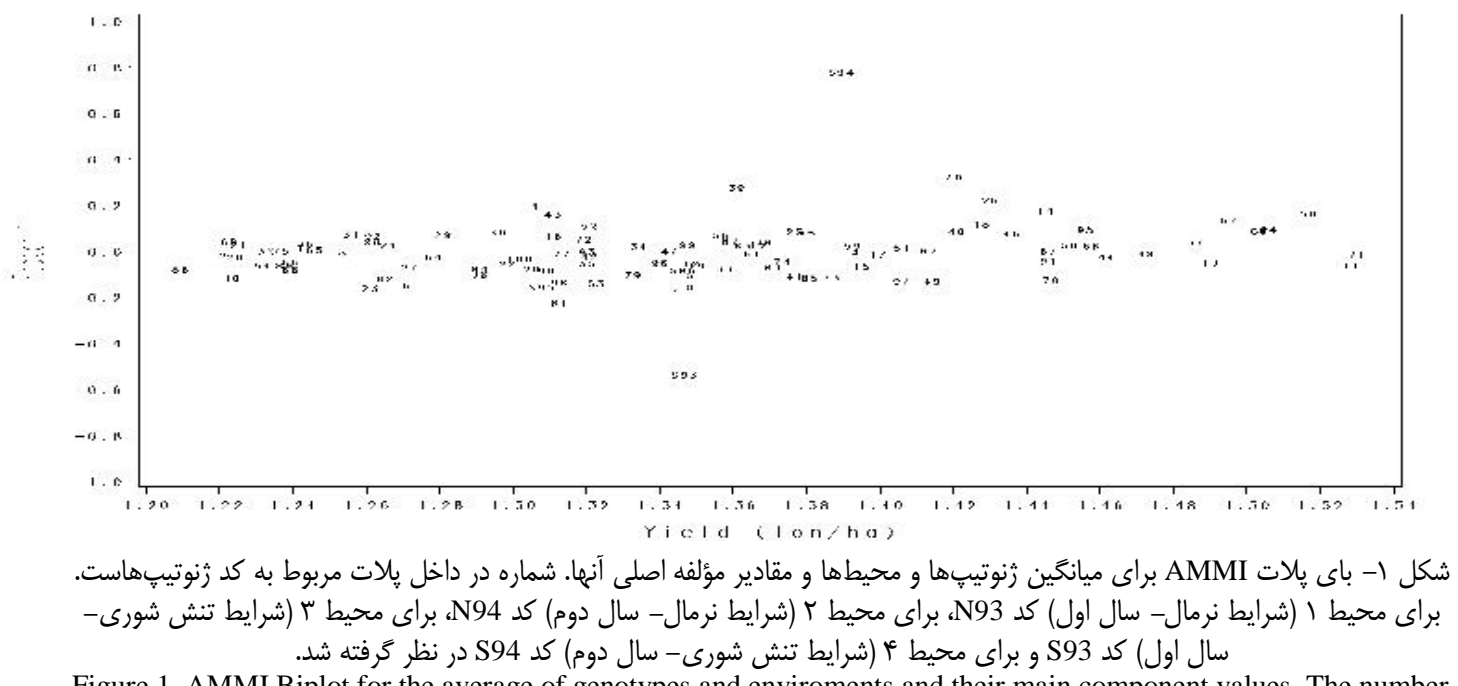

Figure 1. AMMI Biplot for the average of genotypes and enviroments and their main component values. The number inside the plot is the genotype codes. For environment 1 (normal conditions - first year), code of N93, for environment 2 (normal conditions - second year) code of N94, for environment 3 (salt stress conditions - first year) code of S93 and for environment 4 (salt stress conditions - second year) code of S94 was considered.

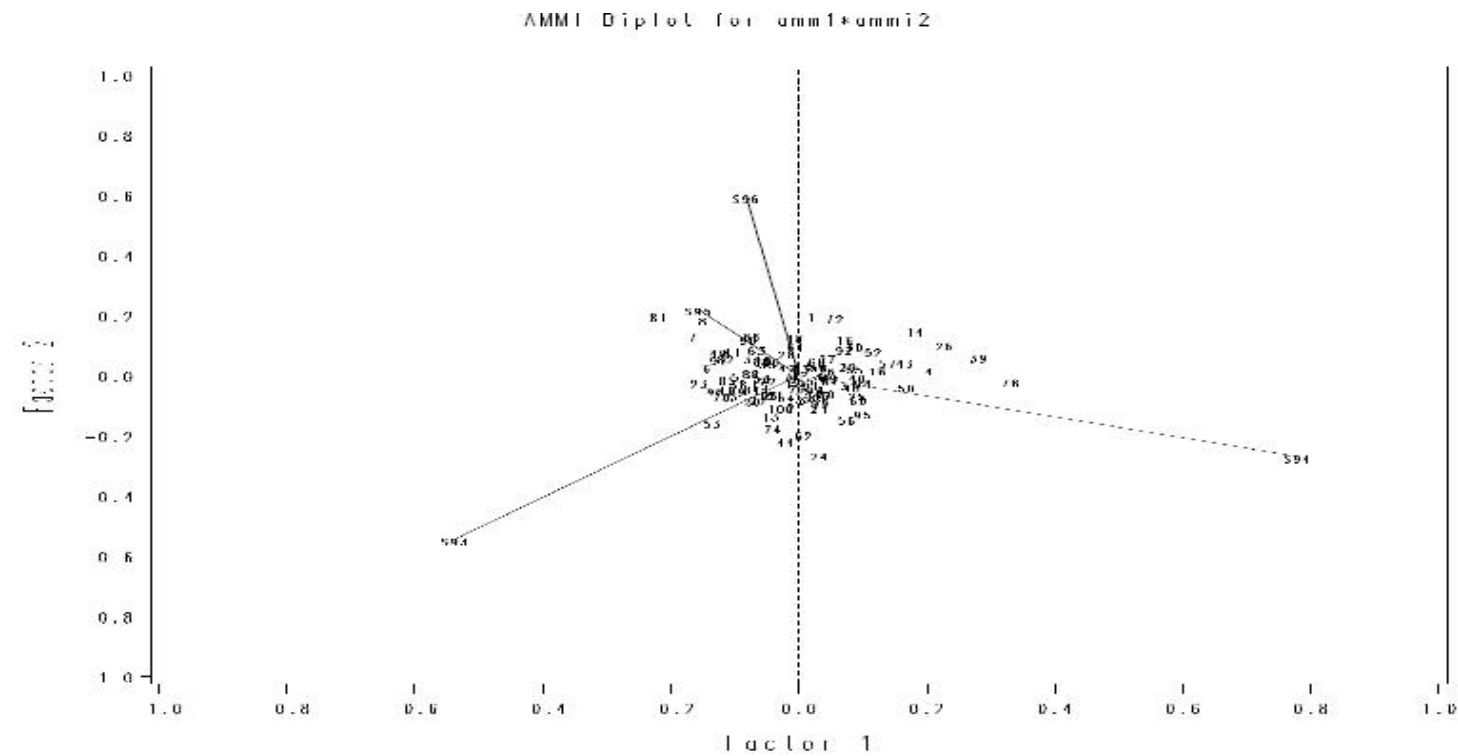

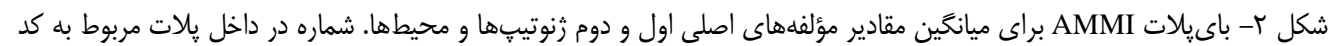

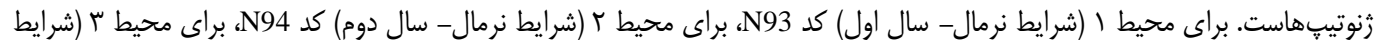

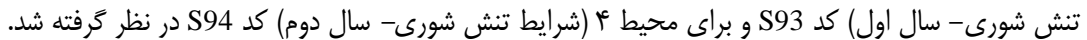

Figure 2. AMMI Biplot for the mean values of first and second principal components of genotypes and environments. The number inside the plot is genotype codes. For environment 1 (normal conditions - first year), code of N93, for environment 2 (normal conditions - second year) code of N94, for environment 3 (salt stress conditions - first year) code of S93 and for environment 4 (salt stress conditions - second year) code of S94 was considered.

بر اساس روش AMMI و ساير آمارههاى يايدارى مورد

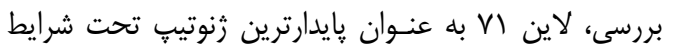

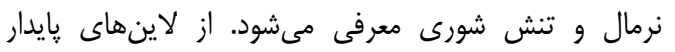

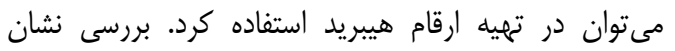

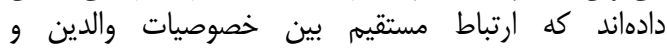
هيبريدهاى حاصل وجود دارد.

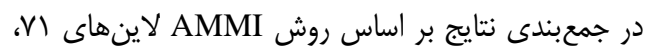

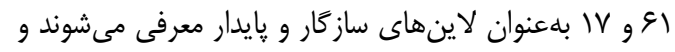

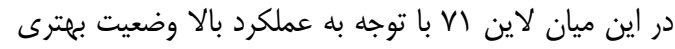

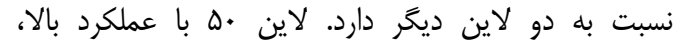

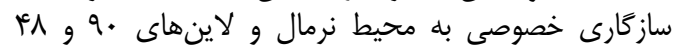
عملكرد بالا و سازگًارى خصوصى بهى به شرايط شور نشان دادند. 
1. Albert, MJ A 2004. A comparison of statical methods to describegenotype $\times$ environment interaction and yield stability in multi-location maizetrials. M.Sc. Thesis, Department of Plant Science, the University of the Free State, Blomfontein, South Africa. $96 \mathrm{pp}$.

2. Akcura, M., Y. Kaya and S. Taner. 2005. Genotype-environment interaction andphenotypic stability analysis for grain yield of durum wheat in the centralAntaolian region. Turkish Journal of Agriculture and Forestry, 29(5): 369-375.

3. Crossa, J., H.G. Gauch and R.W. Zobel. 1990. Additive main effects and multiplicative interaction analysis of two maize cultivar trials. Crop Science, 30(3): 493-500.

4. Deharo, A., M. Del Rio, J.C. Lopez, M.A. Garcia, M.J. Palomares and J. Fernandes Martines. 1997. Evaluation of the world collection of safflower for oil quality and otherseed characters. Sesame Safflower Newsl, 6: 94-99.

5. Eberhart, S.A. and W.A. Russell. 1966. Stability parameters for comparing varieties. Crop Science, 6(1): 36-40.

6. Fattahi, F. and A. Yusefi. 2005. Evaluation of yield stability of barley genotypesusing stability parameters and pattern analysis via AMMI model. Iranian Journal of Agricultural Sciences, 37: 317-326.

7. Finlay, K.W. and G.N. Wilkinson. 1963. The analysis of adaptation in a plant breeding program. Australian Journal of Agricultural Research, 14(6): 742-754.

8. Francis, T.R. and L.W. Kannenberg. 1978. Yield stability studies in short-season maize. Canadian Journal of Plant Science, 58(4): 1029-1034.

9. Farshadfar, E. 2000. Application of Biometrical Genetics in Plant Breeding. Razi University Press, Kermanshah, Iran. 396 pp. (In Persian).

10. Gauch, H.G. 1992. Statistical Analysis of Regional Yield Trials: AMMI Analysis of Factorial Designs. Amsterdam, The Netherlands: Elsevier, $278 \mathrm{pp}$.

11. Guach, H.G. and R.W. Zobel. 1997. Identifying mega environments andtargeting genotypes. Crop Science, 37(2): 311-326.

12. Goncalves, P., N. Bartoletto, R. Martns and G. Gallo. 2003. Genotype-environment interaction and phenotypic stability for girth growth and rubber yield of hevea clones in Sao Paulo state, Brizil. Genetics and MolecularBiology, 26(4): 441-48.

13. Hossain, M.A., L. Rahmand and A.K.M. Shamsuddin. 2003. Genotype environment interaction and stability analysis in soybean. Journal of Biological Sciences, 3(11): 1026-1031.

14. Hatamzadeh, H., A. Khazai and K.H. Alizadeh. 2011. Identification of stable genotypes of rapeseed using some parametric and non-parametric methods under dryland conditions. International Research Journal of Applied and Basic Sciences, 2(1): 73-84.

15. IRRISTAT 4.3 for Windows. 2002. Tutorial Manual, Biometrics Unit. International Rice Research Institute, $182 \mathrm{pp}$.

16. Kadi, Z., F. Adjel and H. Bouzerzour. 2010. Analysis of genotype environment interaction of barley grain yield (Hordeum vulgar L.) under semi arid conditions. Advances in Environmental Biology, 4(1): 34-40.

17. Kaya, Y., M. Akcura and S. Taner. 2006. GGE-Biplot analysis of multi environment yield trials in bread wheat. Turkish Journal of Agriculture and Forestry, 30(6): 325-337.

18. Lin, C. and M.R. Binns. 1991. Genetic properties of four types of stability parameter. Theoretical and Applied Genetics, 82(4): 505-509.

19. Moameni, A., H. Siadat and M.J. Malakouti. 1999. The extent distribution and management of saltaffected soils of Iran. FAO Global Network on Integrated Soil Management for Sustainable Use of Salt affected Soils. Izmir, Turkey.

20. Morsali Aghajari, F., R. Darvishzadeh, H. Hatami Maleki, M. Barin and N. Abbaspour. 2015. Identification of QTLs controlling yield and yield components in sunflower under salinity stress conditions. Iranian Journal of Filed Crop Science, 47(1): 87-98 (In Persian).

21. Nezami, A., H.R. Khazaei, Z. Boroumand Rezazadeh and A. Hosseini. 2008. Effects of drought stress and defoliation on sunflower (Helianthus annuus L.) in controlled conditions. Desert, 12(2): 99-104.

22. Narkhede, B.N. and A.M. Patil. 1990. Phenotypic stability in safflower. Journal of Maharashtra Agricultural University, 15(1): 95-96.

23. Plaisted, R.L. and L.C. Peterson. 1959. A technique for evaluating the ability of selection to yield consistently in different locations and seasons. American Potato Journal, 36(11): 381-385.

24. Purchase, J.L., H. Hatting and C.S. Vandeventer. 2000. Genotypexenvironment interaction of winter wheat (Triticum aestivum L.) in South Africa: II. Stability analysis of yield performance. South African Journal of Plant and Soil, 17(3): 101-107.

25. Pinthus, J.M. 1973. Estimate of genotype value: a proposed method. Euphitica, 22(1): 121-123.

26. Rudra, V., B.N. Harish and S.D. Yaragoppa. 2005. Phenotypic stability for safflower advanced breeding lines under rainfed ecosystem of north Karnataka. VI ${ }^{\text {th }}$ International SafflowerConference, 26-30 pp.

27. Roemer, T. 1917. Sin die ertragsreichen sorten ertragssicherer. Mitt. DLG, 32: 87-89.

28. Shukla, G.K. 1972. Some statistical aspects of partitioning genotype-environmental components of variability. Heredity, 29: 237-245.

29. Schoeman, L.J. 2003. Genotypex environment interaction in sunflower (Helianthus annuus) in south Aefrica. M. Sc. Thesis, Department of Agronomy, University of the Free State, Bloemfontein, South Africa. $200 \mathrm{pp}$.

30. Vashev, B., T. Gaiser, T. Ghawana, A. de Vries and K. Stahr. 2010. Biosafor Project Deliverable 9: Cropping Potentials for Saline Areas in India, Pakistan and Bangladesh. University of Hohenheim, Hohenheim, Germany.

31. Wricke, G. 1962. Uber eine methode zur refassung der okologischen streubretite in feldversuchen. Flazenzuecht, 47: 92-96.

32. Zobel, R.W. and H.G. Gauch. 1988. Statistical analysis of a yield trial. Agronomy Journal, 80(3): 388-393. 


\title{
Evaluation of Yield Stability of Sunflower Inbred Lines under Salt Stress Conditions
}

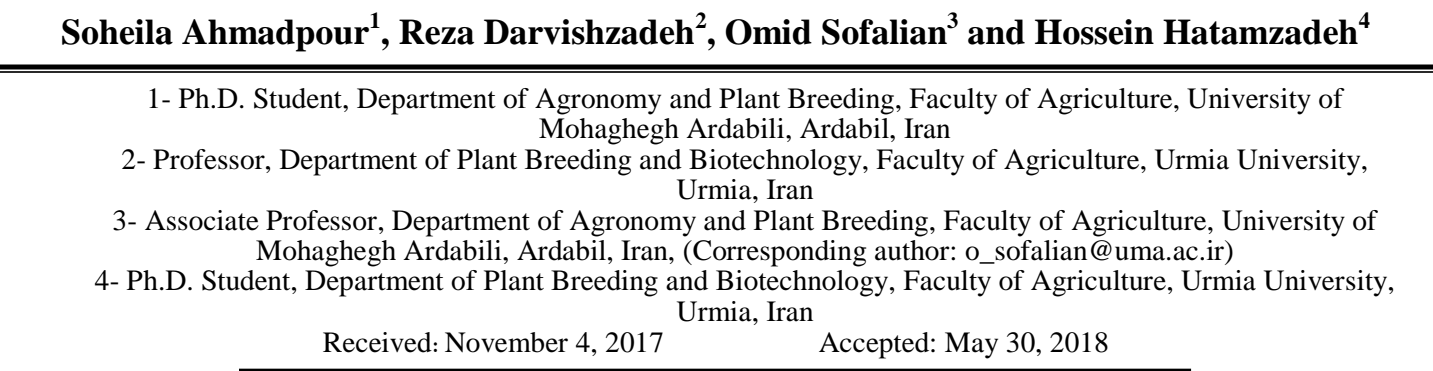

\begin{abstract}
The genotype by environment interaction is a major challenge in the study of quantitative characters because it complicates the interpretation of genetic experiments and do predictions difficult, also it reduces grain seed yield stability in different environments. In order to determine the yield stability, adaptability and analysis of the genotype $\times$ environment interaction of oily sunflower inbred lines under normal and salt stress conditions, 100 genotypes coming from different geographical regions were evaluated using a randomized complete block design with three replications for two successive years (2006 to 2007). In stability analysis using statistics such as environmental variance and coefficient of variation, lines 71 and 33 showed minimum variations compared to other lines. Based on Eberhart and Russell regression method, lines 71, 45, 40 and 25 was the most stable genotypes. AMMI statistics revealed lines with code numbers of 71, 77, 93 and 51 as the most stable genotypes. Biplot technique was used to identify the appropriate genotypes for special environments. Based on this method, lines 71 , 61 and 17 showed the lowest interaction and considered as the most stable genotypes. However, line 71 showed highest seed yield compared two other lines. The line with code number of 50 showed special stability and high yield under normal conditions whereas lines 90, 48 showed special stability and high yield under salt stress conditions. In conclusion, based on different stability analysis statistics and AMMI analysis the line 71 are introduced as most stable line under normal and salt stress conditions.
\end{abstract}

Keywords: Biplot, Genotype by Environment Interaction, Salt Stress, Sunflower 